\title{
PROBABILISTIC METHODS FOR THE INCOMPRESSIBLE NAVIER-STOKES EQUATIONS WITH SPACE PERIODIC CONDITIONS
}

\author{
G. N. MILSTEIN, ${ }^{*}$ Ural Federal University \\ M. V. TRETYAKOV, ${ }^{* *}$ University of Leicester and University of Nottingham
}

\begin{abstract}
We propose and study a number of layer methods for Navier-Stokes equations (NSEs) with spatial periodic boundary conditions. The methods are constructed using probabilistic representations of solutions to NSEs and exploiting ideas of the weak sense numerical integration of stochastic differential equations. Despite their probabilistic nature, the layer methods are nevertheless deterministic.

Keywords: Probabilistic representations of solutions of partial differential equations; Feynman-Kac formula; weak approximation of stochastic differential equations; layer method; Helmholtz-Hodge decomposition
\end{abstract}

2010 Mathematics Subject Classification: Primary 35Q30

Secondary $65 \mathrm{M} 25 ; 65 \mathrm{M} 12 ; 60 \mathrm{H} 30$

\section{Introduction}

The importance of Navier-Stokes equations (NSEs) for various applications is undisputed and there is a significant need for efficient direct numerical simulation of NSEs. The theory and applications of NSEs can be found, in e.g. [3], [4], [11], [13], [30], [31]. The commonly used numerical methods for NSEs include finite element methods (see, e.g. [6], [7], and [8]), finite difference schemes (see, e.g. [6], [7], and [32]), spectral methods (see, e.g. [2], [6], [7], and [26]), multilevel methods [4] and [32]. flow:

We consider the system of NSEs for velocity $v$ and pressure $p$ in a viscous incompressible

$$
\begin{gathered}
\frac{\partial v}{\partial s}+(v, \nabla) v+\nabla p-\frac{\sigma^{2}}{2} \Delta v=f, \\
\operatorname{div} v=0 .
\end{gathered}
$$

In (1.1)-(1.2) we have $-T \leq s \leq 0, x \in \mathbb{R}^{n}, v \in \mathbb{R}^{n}, f \in \mathbb{R}^{n}$, and $p$ is a scalar. The velocity vector $v=\left(v^{1}, \ldots, v^{n}\right)^{\top}$ satisfies the initial conditions

$$
v(-T, x)=\varphi(x)
$$

and the spatial periodic conditions

$$
v\left(s, x+L e_{i}\right)=v(s, x), \quad i=1, \ldots, n,-T \leq s \leq 0 .
$$

Received 17 May 2011; revision received 18 May 2012.

* Postal address: Ural Federal University, Lenin Str. 51, 620083 Ekaterinburg, Russia.

Email address: grigori.milstein@usu.ru

** Postal address: School of Mathematical Sciences, University of Nottingham, Nottingham NG7 2RD, UK.

Email address: michael.tretyakov@nottingham.ac.uk 
Here $\left\{e_{i}\right\}$ is the canonical basis in $\mathbb{R}^{n}$ and $L>0$ is the period. For simplicity in writing, the periods in all the directions are taken the same. The function $f=f(s, x)$ and pressure $p=p(s, x)$ are supposed to be spatially periodic as well. The system of NSEs (1.1) is autonomous and, consequently, its solution does not depend on a time shift. The choice of the interval $[-T, 0]$ is convenient for probabilistic representations of the solution to problem (1.1)-(1.4) after a simple change of variables.

We deal with NSEs in the primitive variable formulation. In this respect we recall [13], [32] that making use of other unknowns, such as the vorticity and stream function, can be advantageous in some special cases, e.g. in two dimensions or when vortices play a key role.

In this paper we propose and study layer methods, which exploit probabilistic representations of solutions to NSEs. We introduce function spaces required, recall the Helmholtz-Hodge decomposition, and give the probabilistic representations in Section 2. Layer methods for semilinear and quasilinear partial differential equations (PDEs) of parabolic type were proposed in [14], [19], and [20] (see also [18]). A layer method for NSEs was first proposed in [1]; specifically, a first-order method which we briefly revise in Section 3.1.

Here we extend application of the probabilistic approach to NSEs in various directions. In Section 3.2 we deal with the approximation of the pressure, which was not considered in [1]. In Sections 3.3 and 3.4 we exploit different probabilistic representations to that used in [1] and propose new layer methods for NSEs. We also establish a relation between layer methods and finite difference schemes. In Section 4 we exploit geometric integration of stochastic differential equations developed in [17], [22], and [23] (see also [18]) in order to construct the corresponding new layer method. We propose and study a second-order method for NSEs in Section 5. In Section 6 we use the results of [15] and [16] to construct a new first-order layer method specifically adapted to the case of small viscosity $\sigma^{2}$. We discuss implementation of the proposed layer methods in Section 7. Results of some numerical experiments on a simple test model of laminar flow from [29] are presented in Section 8.

In this paper we deal with NSEs with periodic boundary conditions (1.1)-(1.4). NSEs with no-slip boundary conditions are treated in [21].

\section{Preliminaries}

In this section we recall the required function spaces [3], [4], [11], [30], [31], [32] and write probabilistic representations of solutions to NSEs.

\subsection{Function spaces and the Helmholtz-Hodge decomposition}

Let $\left\{e_{i}\right\}$ be the canonical basis in $\mathbb{R}^{n}$. We shall consider spatial periodic $n$-vector functions $u(x)=\left(u^{1}(x), \ldots, u^{n}(x)\right)^{\top}$ in $\mathbb{R}^{n}: u\left(x+L e_{i}\right)=u(x), i=1, \ldots, n$, where $L>0$ is the period in the $i$ th direction. Denote by $Q=(0, L)^{n}$ the cube of the period. Of course, one may consider different periods $L_{1}, \ldots, L_{n}$ in the different directions. We denote by $\mathbb{L}^{2}(Q)$ the Hilbert space of functions on $Q$ with the scalar product and the norm

$$
(u, v)=\int_{Q} \sum_{i=1}^{n} u^{i}(x) v^{i}(x) \mathrm{d} x, \quad\|u\|=(u, u)^{1 / 2} .
$$

We keep the notation $|\cdot|$ for the absolute value of numbers and for the length of $n$-dimensional vectors, for example,

$$
|u(x)|=\left[\left(u^{1}(x)\right)^{2}+\cdots+\left(u^{n}(x)\right)^{2}\right]^{1 / 2} .
$$


We denote by $\mathbb{H}_{p}^{m}(Q), m=0,1, \ldots$, the Sobolev space of functions which are in $\mathbb{L}^{2}(Q)$, together with all their derivatives of order less than or equal to $m$, and which are periodic functions with the period $Q$. The space $\mathbb{H}_{p}^{m}(Q)$ is a Hilbert space with the scalar product and the norm

$$
(u, v)_{m}=\int_{Q} \sum_{i=1}^{n} \sum_{\left[\alpha^{i}\right] \leq m} D^{\alpha^{i}} u^{i}(x) D^{\alpha^{i}} v^{i}(x) \mathrm{d} x, \quad\|u\|_{m}=\left[(u, u)_{m}\right]^{1 / 2}
$$

where $\alpha^{i}=\left(\alpha_{1}^{i}, \ldots, \alpha_{n}^{i}\right), \alpha_{j}^{i} \in\{0, \ldots, m\},\left[\alpha^{i}\right]=\alpha_{1}^{i}+\cdots+\alpha_{n}^{i}$, and

$$
D^{\alpha^{i}}=D_{1}^{\alpha_{1}^{i}} \cdots D_{n}^{\alpha_{n}^{i}}=\frac{\partial^{\left[\alpha^{i}\right]}}{\partial\left(x^{1}\right)^{\alpha_{1}^{i}} \ldots \partial\left(x^{n}\right)^{\alpha_{n}^{i}}}, \quad i=1, \ldots, n .
$$

Note that $\mathbb{H}_{p}^{0}(Q)=\mathbb{L}^{2}(Q)$.

Introduce the Hilbert subspaces of $\mathbb{H}_{p}^{m}(Q)$ :

$$
\begin{aligned}
\mathbb{V}_{p}^{m} & =\left\{v: v \in \mathbb{H}_{p}^{m}(Q), \operatorname{div} v=0\right\}, \quad m>0, \\
\mathbb{V}_{p}^{0} & =\text { the closure of } \mathbb{V}_{p}^{m}, \quad m>0 \text { in } \mathbb{L}^{2}(Q) .
\end{aligned}
$$

Clearly,

$$
\mathbb{V}_{p}^{m_{1}}=\text { the closure of } \mathbb{V}_{p}^{m_{2}} \text { in } \mathbb{H}_{p}^{m_{1}}(Q) \text { for any } m_{2} \geq m_{1} .
$$

Denote by $P$ the orthogonal projection in $\mathbb{H}_{p}^{m}(Q)$ onto $\mathbb{V}_{p}^{m}$ (we omit $m$ in the notation $P$ here). The operator $P$ is often called the Leray projection. Due to the Helmholtz-Hodge decomposition, any function $u \in \mathbb{H}_{p}^{m}(Q)$ can be represented as

$$
u=P u+\nabla g, \quad \operatorname{div} P u=0,
$$

where $g=g(x)$ is a scalar $Q$-periodic function such that $\nabla g \in \mathbb{H}_{p}^{m}(Q)$. It is natural to introduce the notation $P^{\perp} u:=\nabla g$ and, hence, write

$$
u=P u+P^{\perp} u
$$

with

$$
P^{\perp} u \in\left(\mathbb{V}_{p}^{m}\right)^{\perp}=\left\{v: v \in \mathbb{H}_{p}^{m}(Q), v=\nabla g\right\}
$$

Let

$$
\begin{gathered}
u(x)=\sum_{\boldsymbol{n} \in \mathbb{Z}^{n}} u_{\boldsymbol{n}} \mathrm{e}^{i(2 \pi / L)(\boldsymbol{n}, x)}, \quad g(x)=\sum_{\boldsymbol{n} \in \mathbb{Z}^{n}} g_{\boldsymbol{n}} \mathrm{e}^{i(2 \pi / L)(\boldsymbol{n}, x)}, \quad g_{\boldsymbol{0}}=0, \\
P u(x)=\sum_{\boldsymbol{n} \in \mathbb{Z}^{n}}(P u)_{\boldsymbol{n}} \mathrm{e}^{i(2 \pi / L)(\boldsymbol{n}, x)}, \quad P^{\perp} u(x)=\nabla g(x)=\sum_{\boldsymbol{n} \in \mathbb{Z}^{n}}\left(P^{\perp} u\right)_{\boldsymbol{n}} \mathrm{e}^{i(2 \pi / L)(\boldsymbol{n}, x)}
\end{gathered}
$$

be the Fourier expansions of $u, g, P u$, and $P^{\perp} u=\nabla g$. Here $u_{\boldsymbol{n}},(P u)_{\boldsymbol{n}}$, and $\left(P^{\perp} u\right)_{\boldsymbol{n}}=(\nabla g)_{\boldsymbol{n}}$ are $n$-dimensional vectors and the $g_{n}$ are scalars. We note that $g_{\mathbf{0}}$ can be any real number, but for definiteness we set $g_{0}=0$. The coefficients $(P u)_{n},\left(P^{\perp} u\right)_{n}$, and $g_{n}$ can be easily expressed in terms of $u_{n}$ :

$$
\begin{gathered}
(P u)_{n}=u_{\boldsymbol{n}}-\frac{u_{\boldsymbol{n}}^{\top} \boldsymbol{n}}{|\boldsymbol{n}|^{2}} \boldsymbol{n}, \quad\left(P^{\perp} u\right)_{\boldsymbol{n}}=\mathrm{i} \frac{2 \pi}{L} g_{\boldsymbol{n}} \boldsymbol{n}=\frac{u_{\boldsymbol{n}}^{\top} \boldsymbol{n}}{|\boldsymbol{n}|^{2}} \boldsymbol{n}, \\
g_{\boldsymbol{n}}=-\mathrm{i} \frac{L}{2 \pi} \frac{u_{\boldsymbol{n}}^{\top} \boldsymbol{n}}{|\boldsymbol{n}|^{2}}, \quad \boldsymbol{n} \neq \mathbf{0}, g_{\mathbf{0}}=0 .
\end{gathered}
$$


We have

$$
\nabla \mathrm{e}^{\mathrm{i}(2 \pi / L)(\boldsymbol{n}, x)}=\boldsymbol{n} \mathrm{e}^{\mathrm{i}(2 \pi / L)(\boldsymbol{n}, x)} \mathrm{i} \frac{2 \pi}{L} ;
$$

hence, $u_{\boldsymbol{n}} \mathrm{e}^{\mathrm{i}(2 \pi / L)(\boldsymbol{n}, x)} \in \mathbb{V}_{p}^{m}$ if and only if $\left(u_{\boldsymbol{n}}, \boldsymbol{n}\right)=0$. It follows from here that the orthogonal basis of the subspace $\left(\mathbb{V}_{p}^{m}\right)^{\perp}$ consists of $\boldsymbol{n} \mathrm{e}^{\mathrm{i}(2 \pi / L)(\boldsymbol{n}, x)}, \boldsymbol{n} \in \mathbb{Z}^{n}, \boldsymbol{n} \neq \mathbf{0}$; and an orthogonal basis of $\mathbb{V}_{p}^{m}$ consists of ${ }_{k} u_{\boldsymbol{n}} \mathrm{e}^{\mathrm{i}(2 \pi / L)(\boldsymbol{n}, x)}, k=1, \ldots, n-1, \boldsymbol{n} \in \mathbb{Z}^{n}$, where, under $\boldsymbol{n} \neq \mathbf{0}$, the vectors ${ }_{k} u_{\boldsymbol{n}}$ are orthogonal to $\boldsymbol{n}$, i.e. $\left({ }_{k} u_{\boldsymbol{n}}, \boldsymbol{n}\right)=0, k=1, \ldots, n-1$, and they are orthogonal among themselves, i.e. $\left({ }_{k} u_{\boldsymbol{n}}, m u_{\boldsymbol{n}}\right)=0, k, m=1, \ldots, n-1, m \neq k$, and, finally, for $\boldsymbol{n}=\mathbf{0}$, the vectors ${ }_{k} u_{\mathbf{0}}, k=1, \ldots, n$, are orthogonal. In particular, in the two-dimensional case $(n=2)$, these bases are correspondingly

$$
\left[\begin{array}{l}
n_{1} \\
n_{2}
\end{array}\right] \mathrm{e}^{\mathrm{i}(2 \pi / L)(\boldsymbol{n}, x)} \quad \text { and } \quad\left[\begin{array}{c}
-n_{2} \\
n_{1}
\end{array}\right] \mathrm{e}^{\mathrm{i}(2 \pi / L)(\boldsymbol{n}, x)}, \quad \boldsymbol{n}=\left(n_{1}, n_{2}\right)^{\top} .
$$

\subsection{Probabilistic representations}

Introducing in (1.1)-(1.2) the new time $t=-s$ and the new function $u(t, x)=v(-t, x)$, $0 \leq t \leq T$, we obtain

$$
\begin{gathered}
\frac{\partial u}{\partial t}+\frac{\sigma^{2}}{2} \Delta u-(u, \nabla) u-\nabla p+f=0, \\
\operatorname{div} u=0, \\
u(T, x)=\varphi(x), \\
u\left(t, x+L e_{i}\right)=u(t, x), \quad i=1, \ldots, n, 0 \leq t \leq T .
\end{gathered}
$$

In what follows we assume that this problem has a unique, sufficiently smooth classical solution (see the corresponding theoretical results in, e.g. [4], [11], [30], and [31]).

Assumption 2.1. We assume that the solution of problem (2.4)-(2.7), $(u(t, x), p(t, x))$ has continuous derivatives with respect to $t$ and $x$ up to some order for $x \in \mathbb{R}^{n}, 0 \leq t \leq T$.

Now we consider some probabilistic representations of the solution to (2.4)-(2.7).

Let $(u(t, x), p(t, x))$ be a solution of problem (2.4)-(2.7). For the function $u(t, x)$, one can use the following probabilistic representation of solutions to the Cauchy problem for equations of parabolic type (see, e.g. [5] and [18]):

$$
u(t, x)=\mathbb{E}\left[\varphi\left(T, X_{t, x}(T)\right) Q_{t, x, 1}(T)+Z_{t, x, 1,0}(T)\right] .
$$

Here $X_{t, x}(s), Q_{t, x, q}(s)$, and $Z_{t, x, y, z}(s), s \geq t$, solve the system of Itô stochastic differential equations

$$
\begin{aligned}
\mathrm{d} X & =(-u(s, X)-\sigma \mu(s, X)) \mathrm{d} s+\sigma \mathrm{d} W(s), \quad X(t)=x, \\
\mathrm{~d} Q & =\mu^{\top}(s, X) Q \mathrm{~d} W(s), \quad Q(t)=q, \\
\mathrm{~d} Z & =(-\nabla p(s, X)+f(s, X)) Q \mathrm{~d} s+F(s, X) Q \mathrm{~d} W(s), \quad Z(t)=z .
\end{aligned}
$$

In (2.8)-(2.11), $W(s)$ is a standard $n$-dimensional Wiener process, $Q$ is a scalar, and $Z$ is an $n$-dimensional column vector; $\mu(s, x)$ is an arbitrary $n$-dimensional spatial periodic vector function and $F(s, x)$ is an arbitrary $(n \times n)$-dimensional spatial periodic matrix function, which are sufficiently smooth. Probabilistic representations (A) and (B) given below and used for constructing layer methods in the next sections are particular cases of representation (2.8)(2.11). 
(A) For $\mu(s, x)=0$ and $F(s, x)=0,(2.8)$ gives the standard probabilistic representation

$$
u(t, x)=\mathbb{E}\left[\varphi\left(X_{t, x}(T)\right)-\int_{t}^{T} \nabla p\left(s, X_{t, x}(s)\right) \mathrm{d} s+\int_{t}^{T} f\left(s, X_{t, x}(s)\right) \mathrm{d} s\right],
$$

where $X_{t, x}(s), s \geq t$, solves the system of stochastic differential equations

$$
\mathrm{d} X=-u(s, X) \mathrm{d} s+\sigma \mathrm{d} W(s), \quad X(t)=x .
$$

Let $0=t_{0}<t_{1}<\cdots<t_{N}=T$ be a uniform partition of the interval [0,T], and let $h=T / N$ (we restrict ourselves to the uniform partition for simplicity only). Clearly, analogously to (2.12), we can write the following local probabilistic representation of the solution to (2.4)-(2.7):

$$
\begin{gathered}
u\left(t_{k}, x\right)=\mathbb{E}\left[u\left(t_{k+1}, X_{t_{k}, x}\left(t_{k+1}\right)\right)-\int_{t_{k}}^{t_{k+1}} \nabla p\left(s, X_{t_{k}, x}(s)\right) \mathrm{d} s\right. \\
\left.+\int_{t_{k}}^{t_{k+1}} f\left(s, X_{t_{k}, x}(s)\right) \mathrm{d} s\right] .
\end{gathered}
$$

Representation (2.12)-(2.13) is used in a number of works (see, e.g. [1], [13], [25], and the references therein).

(B) For $\mu(s, x) \neq 0$ and $F(s, x)=0$, representation (2.8) follows from Girsanov's theorem. In particular, for $\mu(s, x)=-u(s, x) / \sigma$, we have

$$
\begin{aligned}
u(t, x)= & \mathbb{E}\left[\varphi\left(X_{t, x}(T)\right) Q_{t, x, 1}(T)\right] \\
& +\mathbb{E}\left[-\int_{t}^{T} \nabla p\left(s, X_{t, x}(s)\right) Q_{t, x, 1}(s) \mathrm{d} s+\int_{t}^{T} f\left(s, X_{t, x}(s)\right) Q_{t, x, 1}(s) \mathrm{d} s\right],
\end{aligned}
$$

where $X_{t, x}(s)$ and $Q_{t, x, 1}(s), s \geq t\left(Q_{t, x, 1}(s)\right.$ is a scalar), solve the system of stochastic differential equations

$$
\begin{aligned}
\mathrm{d} X & =\sigma \mathrm{d} W(s), \quad X(t)=x, \\
\mathrm{~d} Q & =-\frac{1}{\sigma} Q u^{\top}(s, X) \mathrm{d} W(s), \quad Q(t)=1 .
\end{aligned}
$$

The corresponding local probabilistic representation has the form

$$
\begin{aligned}
u\left(t_{k}, x\right)= & \mathbb{E}\left[u\left(t_{k+1}, X_{t_{k}, x}\left(t_{k+1}\right)\right) Q_{t_{k}, x, 1}\left(t_{k+1}\right)\right] \\
& +\mathbb{E}\left[-\int_{t_{k}}^{t_{k+1}} \nabla p\left(s, X_{t_{k}, x}(s)\right) Q_{t_{k}, x, 1}(s) \mathrm{d} s+\int_{t_{k}}^{t_{k+1}} f\left(s, X_{t_{k}, x}(s)\right) Q_{t_{k}, x, 1}(s) \mathrm{d} s\right] .
\end{aligned}
$$

(C) We can also write the following probabilistic representation of the solution to (2.4)-(2.7):

$$
\begin{aligned}
u(t, x)= & \mathbb{E} \varphi\left(X_{t, x}(T)\right) \\
& -\mathbb{E}\left[\int_{t}^{T}\left(u\left(s, X_{t, x}(s)\right), \nabla\right) u\left(s, X_{t, x}(s)\right) \mathrm{d} s+\int_{t}^{T} \nabla p\left(s, X_{t, x}(s)\right) \mathrm{d} s\right] \\
& +\mathbb{E} \int_{t}^{T} f\left(s, X_{t, x}(s)\right) \mathrm{d} s .
\end{aligned}
$$

Here $X_{t, x}(s), s \geq t$, solves the system of stochastic differential equations

$$
\mathrm{d} X=\sigma \mathrm{d} W(s), \quad X(t)=x .
$$


The corresponding local probabilistic representation has the form

$$
\begin{aligned}
u\left(t_{k}, x\right)= & \mathbb{E} u\left(t_{k+1}, X_{t_{k}, x}\left(t_{k+1}\right)\right) \\
& -\mathbb{E}\left[\int_{t_{k}}^{t_{k+1}}\left(u\left(s, X_{t, x}(s)\right), \nabla\right) u\left(s, X_{t, x}(s)\right) \mathrm{d} s+\int_{t_{k}}^{t_{k+1}} \nabla p\left(s, X_{t_{k}, x}(s)\right) \mathrm{d} s\right] \\
& +\mathbb{E} \int_{t_{k}}^{t_{k+1}} f\left(s, X_{t_{k}, x}(s)\right) \mathrm{d} s .
\end{aligned}
$$

This representation is analogous to that used in [20] for constructing layer methods for quasilinear PDEs.

(D) Introduce the system of forward-backward stochastic differential equations (FBSDEs) [12], [24]:

$$
\begin{aligned}
& \mathrm{d} X=-Y \mathrm{~d} s+\sigma \mathrm{d} W(s), \quad X(t)=x, \\
& \mathrm{~d} Y=(\nabla p(s, X)-f(s, X)) \mathrm{d} s+Z \mathrm{~d} W(s), \quad Y(T)=\varphi\left(X_{t, x}(T)\right) .
\end{aligned}
$$

Here $X, Y, W, f$, and $\nabla p$ are column vectors of dimension $n$ and $Z$ is an $n \times n$ matrix (we note that we use the same letter $Z$ in (2.11) and (2.22) for two different processes, but this does not lead to any confusion). Let $u(t, x)$ be a solution of (2.4) and (2.6). It is straightforward to prove that

$$
X(s)=X_{t, x}(s), \quad Y(s)=u\left(s, X_{t, x}(s)\right), \quad Z^{k j}(s)=\sigma \frac{\partial u^{k}}{\partial x^{j}}\left(s, X_{t, x}(s)\right)
$$

is a solution of (2.22). Conversely, if $(X(s), Y(s), Z(s))$ is a solution of (2.22) then

$$
u(t, x)=Y(t)
$$

is a solution of (2.4), (2.6). Condition (2.5) is satisfied by choosing an appropriate pressure $p$. In addition,

$$
\frac{\partial u^{k}}{\partial x^{j}}(t, x)=\frac{1}{\sigma} Z^{k j}(t) .
$$

One can use not only the FBSDEs (2.22) but also many others, among which is, e.g. the FBSDEs

$$
\begin{aligned}
& \mathrm{d} X=\sigma \mathrm{d} W(s), \quad X(t)=x, \\
& \mathrm{~d} Y=\left(\nabla p(s, X)-f(s, X)+\frac{1}{\sigma} Y Z\right) \mathrm{d} s+Z \mathrm{~d} W(s), \quad Y(T)=\varphi\left(X_{t, x}(T)\right) .
\end{aligned}
$$

The relations between the solutions of (2.4), (2.6), and (2.26) are again given by the equalities (2.23)-(2.25).

Connections between nonlinear PDEs and FBSDEs turned out to be mutually beneficial for their numerics (see, e.g. [19], [20], and the references therein). An additional advantage of such a numerical approach is the possibility of computing derivatives due to (2.25). Let us note that in [9] regression methods for numerical analysis of BSDEs are invoked.

Below we construct a number of numerical methods based on the probabilistic representations (2.12)-(2.13), (2.15)-(2.17), and (2.19)-(2.20). Applications of the approach based on FBSDEs will appear elsewhere. 


\section{First-order methods}

In this section we first revisit the first-order layer method for NSEs derived in [1]. Then in Section 3.2 we consider approximation of the pressure, which was not addressed in [1]. In Section 3.3 we obtain new first-order methods based on the local probabilistic representation (2.16)-(2.18), and establish a relation between layer methods and finite difference schemes. Section 3.4 deals with a new layer method based on the local probabilistic representation (2.20)-(2.21).

\subsection{A revisited first-order method}

A probabilistic approach to constructing layer methods for solving nonlinear PDEs is proposed in [14] (see also [18]). It is based on local probabilistic representations and on the ideas of the weak sense numerical integration of stochastic differential equations. In spite of the probabilistic nature the methods are nevertheless deterministic. Let us recall the firstorder layer method (see [1]) for constructing approximations of $u\left(t_{k}, x\right)$ based on the results of [14].

For clarity of the exposition, we consider here the three-dimensional case although everything in this section is true for any dimension.

Let us fix $k$ for a while and assume that the function $u\left(t_{k+1}, x\right)$, as a function of $x$, is known, i.e. we assume that the solution on the layer $t=t_{k+1}$ is known. A slightly modified explicit Euler scheme with the simplest noise simulation applied to (2.13) gives

$$
X_{t_{k}, x}\left(t_{k+1}\right) \simeq \bar{X}_{t_{k}, x}\left(t_{k+1}\right)=x-u\left(t_{k+1}, x\right) h+\sigma \sqrt{h} \xi,
$$

where $\xi=\left(\xi^{1}, \xi^{2}, \xi^{3}\right)^{\top}$ and $\xi^{1}, \xi^{2}, \xi^{3}$ are independent and identically distributed (i.i.d.) random variables with the law $\mathbb{P}\left(\xi^{i}= \pm 1\right)=\frac{1}{2}, i=1,2,3$. We substitute $\bar{X}_{t_{k}, x}\left(t_{k+1}\right)$ from (3.1) into (2.14) instead of $X_{t_{k}, x}\left(t_{k+1}\right)$, evaluate the expectation exactly, and thus obtain

$$
\begin{aligned}
u\left(t_{k}, x\right)= & v\left(t_{k}, x\right)-\nabla p\left(t_{k}, x\right) h+f\left(t_{k}, x\right) h+O\left(h^{2}\right) \\
= & P v\left(t_{k}, x\right)+P f\left(t_{k}, x\right) h+P^{\perp} v\left(t_{k}, x\right)+P^{\perp} f\left(t_{k}, x\right) h \\
& -\nabla p\left(t_{k}, x\right) h+O\left(h^{2}\right),
\end{aligned}
$$

where

$$
v\left(t_{k}, x\right)=\mathbb{E} u\left(t_{k+1}, \bar{X}_{k+1}\right)=\frac{1}{8} \sum_{q=1}^{8} u\left(t_{k+1}, x-u\left(t_{k+1}, x\right) h+\sigma \sqrt{h} \xi_{q}\right)
$$

and $\xi_{1}=(1,1,1)^{\top}, \ldots, \xi_{8}=(-1,-1,-1)^{\top}$. Taking into account the fact that $u\left(t_{k}, x\right)$ in (3.2) is divergence free, we obtain

$$
u\left(t_{k}, x\right)=P v\left(t_{k}, x\right)+P f\left(t_{k}, x\right) h+O\left(h^{2}\right) .
$$

The remainders $O\left(h^{2}\right)$ in (3.2) and in (3.4) are functions of $k, x$, and $h$ and they are of the second order of smallness in $h$ in the sense of the space $\mathbb{L}^{2}(Q)$, i.e. $\left\|O\left(h^{2}\right)\right\| \leq K h^{2}$, where $K>0$ does not depend on $k$ and $h$ (see a deterministic proof of this one-step error estimate within Proposition 3.1 on the global error below). Neglecting the remainders, we get the one-step approximations for $u\left(t_{k}, x\right)$ and $\nabla p\left(t_{k}, x\right) h$. The corresponding layer method proposed in [1] (we note that $f=0$ in [1]) has the form

$$
\begin{gathered}
\bar{u}\left(t_{N}, x\right)=\varphi(x), \quad \bar{u}\left(t_{k}, x\right)=P \bar{v}\left(t_{k}, x\right)+P f\left(t_{k}, x\right) h, \\
\nabla \bar{p}\left(t_{k}, x\right) h=P^{\perp} \bar{v}\left(t_{k}, x\right)+P^{\perp} f\left(t_{k}, x\right) h, \quad k=N-1, \ldots, 0,
\end{gathered}
$$


where

$$
\bar{v}\left(t_{k}, x\right)=\frac{1}{8} \sum_{q=1}^{8} \bar{u}\left(t_{k+1}, x-\bar{u}\left(t_{k+1}, x\right) h+\sigma \sqrt{h} \xi_{q}\right) .
$$

Knowing the expansions

$$
\bar{v}\left(t_{k}, x\right)=\sum_{\boldsymbol{n} \in \mathbb{Z}^{3}} \bar{v}_{\boldsymbol{n}} \mathrm{e}^{\mathrm{i}(2 \pi / L)(\boldsymbol{n}, x)}, \quad f\left(t_{k}, x\right)=\sum_{\boldsymbol{n} \in \mathbb{Z}^{3}} f_{\boldsymbol{n}} \mathrm{e}^{\mathrm{i}(2 \pi / L)(\boldsymbol{n}, x)},
$$

it is not difficult to find $\bar{u}\left(t_{k}, x\right)$ and $\bar{p}\left(t_{k}, x\right)$. Indeed, using (2.1) and (2.2), we obtain, from (3.5)-(3.6),

$$
\begin{gathered}
\bar{u}\left(t_{k}, x\right)=\sum_{\boldsymbol{n} \in \mathbb{Z}^{3}} \bar{u}_{\boldsymbol{n}} \mathrm{e}^{\mathrm{i}(2 \pi / L)(\boldsymbol{n}, x)}, \quad \bar{u}_{\boldsymbol{n}}=\bar{v}_{\boldsymbol{n}}+f_{\boldsymbol{n}} h-\frac{\bar{v}_{\boldsymbol{n}}^{\top} \boldsymbol{n}}{|\boldsymbol{n}|^{2}} \boldsymbol{n}-h \frac{f_{\boldsymbol{n}}^{\top} \boldsymbol{n}}{|\boldsymbol{n}|^{2}} \boldsymbol{n}, \\
\bar{p}\left(t_{k}, x\right) h=h \sum_{\boldsymbol{n} \in \mathbb{Z}^{3}} \bar{p}_{\boldsymbol{n}} \mathrm{e}^{\mathrm{i}(2 \pi / L)(\boldsymbol{n}, x)}, \\
\bar{p}_{\boldsymbol{n}} h=-\mathrm{i} \frac{L}{2 \pi}\left(\frac{\bar{v}_{\boldsymbol{n}}^{\top} \boldsymbol{n}}{|\boldsymbol{n}|^{2}}+h \frac{f_{\boldsymbol{n}}^{\top} \boldsymbol{n}}{|\boldsymbol{n}|^{2}}\right), \quad \boldsymbol{n} \neq \mathbf{0}, \bar{p}_{\mathbf{0}}=0 .
\end{gathered}
$$

For the sake of simplicity, the dependences on $k$ are omitted in the notation for the coefficients in (3.8).

It follows from (3.2)-(3.3) that the one-step approximation for $u\left(t_{k}, x\right)$ of method (3.5)(3.7) is of second order. Then, heuristically, the method converges and it is of first order. In [1] the following proposition is proved (for completeness of the exposition, we present that proof here).

Proposition 3.1. Let Assumption 2.1 hold. Let

$$
\left|\bar{u}\left(t_{k}, x\right)\right| \leq K, \quad\left|\frac{\partial \bar{u}\left(t_{k}, x\right)}{\partial x^{i}}\right| \leq K,
$$

where $K>0$ is independent of $x, h$, and $k$. Then method (3.5), (3.7) is of first order with respect to the velocity $u$, i.e.

$$
\left\|\bar{u}\left(t_{k}, \cdot\right)-u\left(t_{k}, \cdot\right)\right\| \leq C h,
$$

where the constant $C$ does not depend on $h$ and $k$.

Proof. We start with estimating the one-step error of method (3.5). This error on the $k$ th layer (on the $(N-k)$ th step) is equal to $P v\left(t_{k}, x\right)+P f\left(t_{k}, x\right) h-u\left(t_{k}, x\right)$ provided that $\bar{u}\left(t_{k+1}, x\right)=u\left(t_{k+1}, x\right)$ :

$$
\begin{aligned}
P v\left(t_{k}, x\right) & +P f\left(t_{k}, x\right) h-u\left(t_{k}, x\right) \\
= & v\left(t_{k}, x\right)-P^{\perp} v\left(t_{k}, x\right)+P f\left(t_{k}, x\right) h-u\left(t_{k}, x\right) \\
= & \frac{1}{8} \sum_{q=1}^{8} u\left(t_{k+1}, x-u\left(t_{k+1}, x\right) h+\sigma \sqrt{h} \xi_{q}\right)-P^{\perp} v\left(t_{k}, x\right)+f\left(t_{k}, x\right) h \\
& -P^{\perp} f\left(t_{k}, x\right) h-u\left(t_{k}, x\right) .
\end{aligned}
$$


Using Assumption 2.1, we expand the function $u\left(t_{k+1}, x-u\left(t_{k+1}, x\right) h+\sigma \sqrt{h} \xi_{q}\right)$ at $\left(t_{k}, x\right)$ in powers of $h$ and $-u^{j}\left(t_{k+1}, x\right) h+\sigma \sqrt{h} \xi_{q}^{j}, j=1,2,3$, and we find that the terms with $\sqrt{h}$ and $h \sqrt{h}$ in the sum $\sum_{q=1}^{8} u\left(t_{k+1}, x-u\left(t_{k+1}, x\right) h+\sigma \sqrt{h} \xi_{q}\right)$ are annihilated. We obtain

$$
\begin{aligned}
& \frac{1}{8} \sum_{q=1}^{8} u\left(t_{k+1}, x-u\left(t_{k+1}, x\right) h+\sigma \sqrt{h} \xi_{q}\right) \\
& \quad=u\left(t_{k}, x\right)+\frac{\partial u}{\partial t}\left(t_{k}, x\right) h-(u, \nabla) u\left(t_{k}, x\right) h+\frac{1}{2} \sigma^{2} \Delta u\left(t_{k}, x\right) h+r(x, h ; k),
\end{aligned}
$$

where

$$
|r(x, h ; k)| \leq C h^{2}
$$

with $C$ being independent of $x, h$, and $k$. Since $u(t, x)$ solves (2.4), we get, from (3.11) and (3.12),

$$
\begin{aligned}
& P v\left(t_{k}, x\right)+P f\left(t_{k}, x\right) h-u\left(t_{k}, x\right) \\
& \quad=\nabla p\left(t_{k}, x\right) h-P^{\perp} v\left(t_{k}, x\right)-P^{\perp} f\left(t_{k}, x\right) h+r(x, h ; k) .
\end{aligned}
$$

Using the orthogonality of $P v\left(t_{k}, x\right)+P f\left(t_{k}, x\right) h-u\left(t_{k}, x\right)$ and $\nabla p\left(t_{k}, x\right) h-P^{\perp} v\left(t_{k}, x\right)-$ $P^{\perp} f\left(t_{k}, x\right) h$ in $\mathbb{H}_{p}^{0}(Q)$ (we recall that $\operatorname{div}\left(P v\left(t_{k}, x\right)+P f\left(t_{k}, x\right) h-u\left(t_{k}, x\right)\right)=0$ ), we arrive at the estimate for the one-step error:

$$
\left\|P v\left(t_{k}, \cdot\right)+P f\left(t_{k}, \cdot\right) h-u\left(t_{k}, \cdot\right)\right\| \leq C h^{2} .
$$

Here the constant $C$ does not depend on $h$ and $k$.

Now we will prove the global error estimate (3.10). Denote the error of method (3.5), (3.7) on the $k$ th layer as $\varepsilon\left(t_{k}, x\right):=\bar{u}\left(t_{k}, x\right)-u\left(t_{k}, x\right)$. Thus, we have

$$
\bar{u}\left(t_{k}, x\right)=u\left(t_{k}, x\right)+\varepsilon\left(t_{k}, x\right), \quad \bar{u}\left(t_{k+1}, x\right)=u\left(t_{k+1}, x\right)+\varepsilon\left(t_{k+1}, x\right) .
$$

Due to (3.5) and (3.7), we obtain

$$
\begin{aligned}
u\left(t_{k}, x\right)+\varepsilon\left(t_{k}, x\right)= & \bar{u}\left(t_{k}, x\right) \\
= & P \bar{v}\left(t_{k}, x\right)+\operatorname{Pf}\left(t_{k}, x\right) h \\
= & \frac{1}{8} \sum_{q=1}^{8} P\left[\bar{u}\left(t_{k+1}, x-\bar{u}\left(t_{k+1}, x\right) h+\sigma \sqrt{h} \xi_{q}\right)\right]+P f\left(t_{k}, x\right) h \\
= & \frac{1}{8} \sum_{q=1}^{8} P\left[u\left(t_{k+1}, x-\bar{u}\left(t_{k+1}, x\right) h+\sigma \sqrt{h} \xi_{q}\right)\right]+P f\left(t_{k}, x\right) h \\
& +\frac{1}{8} \sum_{q=1}^{8} P\left[\varepsilon\left(t_{k+1}, x-\bar{u}\left(t_{k+1}, x\right) h+\sigma \sqrt{h} \xi_{q}\right)\right] .
\end{aligned}
$$

Using Assumption 2.1, we obtain

$$
u\left(t_{k+1}, x-\bar{u}\left(t_{k+1}, x\right) h+\sigma \sqrt{h} \xi_{q}\right)=u\left(t_{k+1}, x-u\left(t_{k+1}, x\right) h+\sigma \sqrt{h} \xi_{q}\right)+r_{k q}(x),
$$

where

$$
\begin{aligned}
\left|r_{k q}(x)\right| & =\left|u\left(t_{k+1}, x-\bar{u}\left(t_{k+1}, x\right) h+\sigma \sqrt{h} \xi_{q}\right)-u\left(t_{k+1}, x-u\left(t_{k+1}, x\right) h+\sigma \sqrt{h} \xi_{q}\right)\right| \\
& \leq K\left|\varepsilon\left(t_{k+1}, x\right)\right| h .
\end{aligned}
$$


It follows from (3.15) and (3.16) that

$$
\begin{aligned}
u\left(t_{k}, x\right)+\varepsilon\left(t_{k}, x\right)= & \frac{1}{8} \sum_{q=1}^{8} P\left[u\left(t_{k+1}, x-u\left(t_{k+1}, x\right) h+\sigma \sqrt{h} \xi_{q}\right)\right]+\frac{1}{8} \sum_{q=1}^{8} \operatorname{Pr}_{k q}(x) \\
& +P f\left(t_{k}, x\right) h+\frac{1}{8} \sum_{q=1}^{8} P\left[\varepsilon\left(t_{k+1}, x-\bar{u}\left(t_{k+1}, x\right) h+\sigma \sqrt{h} \xi_{q}\right)\right] .
\end{aligned}
$$

Due to (3.3) and (3.14), we obtain

$$
\begin{aligned}
& \frac{1}{8} \sum_{q=1}^{8} P\left[u\left(t_{k+1}, x-u\left(t_{k+1}, x\right) h+\sigma \sqrt{h} \xi_{q}\right)\right]+P f\left(t_{k}, x\right) h \\
& \quad=P v\left(t_{k}, x\right)+P f\left(t_{k}, x\right) h \\
& \quad=u\left(t_{k}, x\right)+\nabla p\left(t_{k}, x\right) h-P^{\perp} v\left(t_{k}, x\right)-P^{\perp} f\left(t_{k}, x\right) h+r(x, h ; k) .
\end{aligned}
$$

Application of the operator $P$ to the left-hand side of (3.19) does not change it, while its application to the right-hand side results in the terms from $\left(\mathbb{V}_{p}^{0}\right)^{\perp}$ being canceled. Consequently, we arrive at

$$
\frac{1}{8} \sum_{q=1}^{8} P\left[u\left(t_{k+1}, x-u\left(t_{k+1}, x\right) h+\sigma \sqrt{h} \xi_{q}\right)\right]+\operatorname{Pf}\left(t_{k}, x\right) h=u\left(t_{k}, x\right)+\operatorname{Pr}(x, h ; k),
$$

where $\|\operatorname{Pr}(x, h ; k)\| \leq\|r(x, h ; k)\| \leq C h^{2}$ due to (3.13). We obtain, from (3.18) and (3.20),

$$
\varepsilon\left(t_{k}, x\right)=\frac{1}{8} \sum_{q=1}^{8} P\left[\varepsilon\left(t_{k+1}, x-\bar{u}\left(t_{k+1}, x\right) h+\sigma \sqrt{h} \xi_{q}\right)\right]+\frac{1}{8} \sum_{q=1}^{8} \operatorname{Pr}_{k q}(x)+O\left(h^{2}\right),
$$

where $\left\|O\left(h^{2}\right)\right\| \leq C h^{2}$.

Now introduce

$$
\varepsilon_{k}:=\left\|\varepsilon\left(t_{k}, \cdot\right)\right\| .
$$

Let us evaluate the norm $\|\cdot\|$ of the function $\delta(x):=\varepsilon\left(t_{k+1}, x-\bar{u}\left(t_{k+1}, x\right) h+\sigma \sqrt{h} \xi_{q}\right)$. We have

$$
\begin{aligned}
\|\delta\|^{2} & =\int_{Q} \sum_{i=1}^{3}\left[\varepsilon^{i}\left(t_{k+1}, x-\bar{u}\left(t_{k+1}, x\right) h+\sigma \sqrt{h} \xi_{q}\right)\right]^{2} \mathrm{~d} x \\
& =\int_{Q} \sum_{i=1}^{3}\left[\varepsilon^{i}\left(t_{k+1}, y\right)\right]^{2} \frac{D\left(x^{1}, x^{2}, x^{3}\right)}{D\left(y^{1}, y^{2}, y^{3}\right)} \mathrm{d} y,
\end{aligned}
$$

where

$$
y^{j}=x^{j}-\bar{u}^{j}\left(t_{k+1}, x\right) h+\sigma \sqrt{h} \xi_{q}^{j}, \quad j=1,2,3 .
$$

Due to condition (3.9) on the uniform boundedness of $\partial \bar{u}^{i} / \partial x^{j}$ and the fact that $\operatorname{div} \bar{u}=0$, we obtain

$$
\frac{D\left(y^{1}, y^{2}, y^{3}\right)}{D\left(x^{1}, x^{2}, x^{3}\right)}=\left|\begin{array}{ccc}
1-\frac{h \partial \bar{u}^{1}}{\partial x^{1}} & -\frac{h \partial \bar{u}^{1}}{\partial x^{2}} & -\frac{h \partial \bar{u}^{1}}{\partial x^{3}} \\
-\frac{h \partial \bar{u}^{2}}{\partial x^{1}} & 1-\frac{h \partial \bar{u}^{2}}{\partial x^{2}} & -\frac{h \partial \bar{u}^{2}}{\partial x^{3}} \\
-\frac{h \partial \bar{u}^{3}}{\partial x^{1}} & -\frac{h \partial \bar{u}^{3}}{\partial x^{2}} & 1-\frac{h \partial \bar{u}^{3}}{\partial x^{3}}
\end{array}\right|=1+O\left(h^{2}\right)
$$


Therefore,

$$
\|\delta\| \leq \varepsilon_{k+1}\left(1+C h^{2}\right) .
$$

Since $\|P\| \leq 1$, we obtain, from (3.17) and (3.21) (in addition we recall that $\varepsilon\left(t_{N}, x\right)=0$ ),

$$
\varepsilon_{N}=0, \quad \varepsilon_{k} \leq \varepsilon_{k+1}+K \varepsilon_{k+1} h+C h^{2}, \quad k=N-1, \ldots, 1,0 .
$$

Consequently,

$$
\varepsilon_{k} \leq \frac{C}{K}\left(\mathrm{e}^{K T}-1\right) h, \quad k=N, \ldots, 0 .
$$

This completes the proof.

Remark 3.1. We have not succeeded in deriving the inequalities in (3.9) for the approximate solution. At the same time, verifying (3.9) in numerical experiments is straightforward. We also note that in the case of the Oseen-Stokes equations

$$
\frac{\partial v}{\partial s}+(a, \nabla) v+\nabla p-\frac{\sigma^{2}}{2} \Delta v=f
$$

with conditions (1.2)-(1.4), where $a=a(t, x)$ is an $n$-dimensional vector, we succeeded in deriving such inequalities.

\subsection{Approximating pressure}

Approximation of the pressure $p$ was not considered in [1]. Let us proceed to this problem. It follows from (3.2) that the one-step error of $\nabla p\left(t_{k}, x\right) h$ by (3.6) is $O\left(h^{2}\right)$; hence, the one-step error of $\nabla p\left(t_{k}, x\right)$ is $O(h)$. That is why a proof of the convergence of method (3.6) requires additional arguments. To this end, let us make use of the well-known equation

$$
\Delta p\left(t_{k}, x\right)=\operatorname{div} f\left(t_{k}, x\right)-\sum_{i, j=1}^{3} \frac{\partial u^{j}}{\partial x^{i}}\left(t_{k}, x\right) \frac{\partial u^{i}}{\partial x^{j}}\left(t_{k}, x\right) .
$$

In [19] we constructed, in particular, first-order approximate solutions $\bar{u}$ to semilinear parabolic equations by layer methods using a probabilistic approach. We proved there that the derivatives $\partial \bar{u} / \partial x^{i}$ approximate $\partial u / \partial x^{i}$ with the order 1 as well. In all probability such a fact is correct here. If it is so, we can easily solve (see, e.g. [30, p. 10]) the equation

$$
\Delta \tilde{p}\left(t_{k}, x\right)=\operatorname{div} f\left(t_{k}, x\right)-\sum_{i, j=1}^{3} \frac{\partial \bar{u}^{j}}{\partial x^{i}}\left(t_{k}, x\right) \frac{\partial \bar{u}^{i}}{\partial x^{j}}\left(t_{k}, x\right) .
$$

Indeed,

$$
\begin{gathered}
\tilde{p}\left(t_{k}, x\right)=\sum_{\boldsymbol{n} \in \mathbb{Z}^{3}} \tilde{p}_{\boldsymbol{n}} \mathrm{e}^{\mathrm{i}(2 \pi / L)(\boldsymbol{n}, x)}, \\
\tilde{p}_{\boldsymbol{n}}=-\mathrm{i} \frac{L}{2 \pi} \frac{f_{\boldsymbol{n}}^{\top} \boldsymbol{n}}{|\boldsymbol{n}|^{2}}-\frac{1}{|\boldsymbol{n}|^{2}} \sum_{\boldsymbol{m}, \boldsymbol{l} \in \mathbb{Z}^{3}, \boldsymbol{m}+\boldsymbol{l}=\boldsymbol{n}} \bar{u}_{\boldsymbol{m}}^{\top} \boldsymbol{l} \cdot \bar{u}_{\boldsymbol{l}}^{\top} \boldsymbol{m}, \quad \boldsymbol{n} \neq 0, \tilde{p}_{\mathbf{0}}=0 .
\end{gathered}
$$

As a result, we obtain $\tilde{p}\left(t_{k}, x\right)$ which approximates $p\left(t_{k}, x\right)$ with order 1 . It is also possible to prove in this case (i.e. assuming that the derivatives $\partial \bar{u} / \partial x^{i}$ approximate $\partial u / \partial x^{i}$ with the order 1) that $\bar{p}\left(t_{k}, x\right)$ from (3.6) (see in addition (3.8)) approximates $p\left(t_{k}, x\right)$ with order 1 as well. 
The following result (of a less order of accuracy however) is rigorously proved, without assuming that the derivatives $\partial \bar{u} / \partial x^{i}$ approximate $\partial u / \partial x^{i}$ with the order 1 .

Proposition 3.2. Let Assumption 2.1 hold. Let

$$
\left|\bar{u}\left(t_{k}, x\right)\right| \leq K, \quad\left|\frac{\partial \bar{u}\left(t_{k}, x\right)}{\partial x^{i}}\right| \leq K, \quad\left|\frac{\partial^{2} \bar{u}\left(t_{k}, x\right)}{\partial x^{i} \partial x^{j}}\right| \leq K,
$$

where $K>0$ is independent of $x, h$, and $k$. Then $\bar{p}\left(t_{k}, x\right)$ from (3.6) satisfies the inequality

$$
\left\|\bar{p}\left(t_{k}, \cdot\right)-p\left(t_{k}, \cdot\right)\right\| \leq C h^{2 / 3},
$$

where the constant $C$ does not depend on $h$ and $k$.

Proof. Using (3.26), the error estimate (3.10) from Proposition 3.1, and Assumption 2.1, we obtain

$$
\begin{aligned}
\frac{\partial \bar{u}^{i}}{\partial x^{j}}\left(t_{k}, x\right) & =\frac{\bar{u}^{i}\left(t_{k}, x+\gamma h^{1 / 3} e_{j}\right)-\bar{u}^{i}\left(t_{k}, x-\gamma h^{1 / 3} e_{j}\right)}{2 \gamma h^{1 / 3}}+O\left(h^{2 / 3}\right) \\
& =\frac{u^{i}\left(t_{k}, x+\gamma h^{1 / 3} e_{j}\right)-u^{i}\left(t_{k}, x-\gamma h^{1 / 3} e_{j}\right)+O(h)}{2 \gamma h^{1 / 3}}+O\left(h^{2 / 3}\right) \\
& =\frac{\partial u^{i}}{\partial x^{j}}\left(t_{k}, x\right)+O\left(h^{2 / 3}\right)
\end{aligned}
$$

where $\gamma$ is a positive number. It follows from (3.28) that

$$
\left\|\frac{\partial \bar{u}^{i}}{\partial x^{j}}\left(t_{k}, \cdot\right)-\frac{\partial u^{i}}{\partial x^{j}}\left(t_{k}, \cdot\right)\right\| \leq C h^{2 / 3} .
$$

We have, from (3.2) (since $\operatorname{div} u\left(t_{k}, x\right)=0$, i.e. $P^{\perp} u\left(t_{k}, x\right)=0$ ),

$$
\nabla p\left(t_{k}, x\right) h=P^{\perp} \nabla p\left(t_{k}, x\right) h=P^{\perp} v\left(t_{k}, x\right)+P^{\perp} f\left(t_{k}, x\right) h+O\left(h^{2}\right),
$$

and, from (3.6),

$$
\nabla \bar{p}\left(t_{k}, x\right) h=P^{\perp} \nabla \bar{p}\left(t_{k}, x\right) h=P^{\perp} \bar{v}\left(t_{k}, x\right)+P^{\perp} f\left(t_{k}, x\right) h .
$$

Hence,

$$
\nabla\left(p\left(t_{k}, x\right)-\bar{p}\left(t_{k}, x\right)\right) h=P^{\perp}\left(v\left(t_{k}, x\right)-\bar{v}\left(t_{k}, x\right)\right)+O\left(h^{2}\right) .
$$

Furthermore, using (3.3), (3.7), Assumption 2.1, (3.26), and

$$
\operatorname{div} u\left(t_{k+1}, x+\sigma \sqrt{h} \xi_{q}\right)=0, \quad \operatorname{div} \bar{u}\left(t_{k+1}, x+\sigma \sqrt{h} \xi_{q}\right)=0,
$$

we obtain

$$
\begin{gathered}
P^{\perp}\left(v\left(t_{k}, x\right)-\bar{v}\left(t_{k}, x\right)\right) \\
=\frac{1}{8} \sum_{q=1}^{8} P^{\perp}\left[u\left(t_{k+1}, x-u\left(t_{k+1}, x\right) h+\sigma \sqrt{h} \xi_{q}\right)-\bar{u}\left(t_{k+1}, x-\bar{u}\left(t_{k+1}, x\right) h+\sigma \sqrt{h} \xi_{q}\right)\right] \\
=-\frac{h}{8} \sum_{q=1}^{8} \sum_{j} P^{\perp}\left[u^{j}\left(t_{k+1}, x\right) \frac{\partial}{\partial x^{j}} u\left(t_{k+1}, x+\sigma \sqrt{h} \xi_{q}\right)\right. \\
\left.-\bar{u}^{j}\left(t_{k+1}, x\right) \frac{\partial}{\partial x^{j}} \bar{u}\left(t_{k+1}, x+\sigma \sqrt{h} \xi_{q}\right)\right]+O\left(h^{2}\right),
\end{gathered}
$$


where $\left\|O\left(h^{2}\right)\right\| \leq K h^{2}$. From (3.30), (3.31), (3.10), and (3.29), we obtain

$$
\left\|\nabla\left(p\left(t_{k}, x\right)-\bar{p}\left(t_{k}, x\right)\right)\right\| \leq K h^{2 / 3},
$$

whence (3.27) follows.

We observe that we have two ways of finding the pressure: either as a result of projection (3.8) or via solution (3.25) of problem (3.24). Computationally, the second way is slightly more expensive, but one can expect that it results in a more accurate approximation of the pressure, though both approximations are of the same order of accuracy in $h$.

\subsection{New first-order methods based on the probabilistic representation (2.16)-(2.18)}

Using the probabilistic representation (2.16)-(2.18), we consider three new first-order methods. It is interesting that two of them are closely related to standard finite difference schemes.

The first of these layer methods is based on a slightly modified explicit Euler scheme with the simplest noise simulation applied to (2.16)-(2.17), i.e.

$$
\bar{X}_{t_{k}, x}\left(t_{k+1}\right)=x+\sigma \sqrt{h} \xi, \quad \bar{Q}_{t_{k}, x, 1}\left(t_{k+1}\right)=1-\frac{1}{\sigma} u^{\top}\left(t_{k+1}, x\right) \sqrt{h} \xi,
$$

where $\xi$ is the same as in (3.1). Approximating $X_{t_{k}, x}\left(t_{k+1}\right)$ and $Q_{t_{k}, x, 1}\left(t_{k+1}\right)$ in (2.18) by $\bar{X}_{t_{k}, x}\left(t_{k+1}\right)$ and $\bar{Q}_{t_{k}, x, 1}\left(t_{k+1}\right)$ from (3.32), we obtain

$$
\begin{aligned}
u\left(t_{k}, x\right)= & \mathbb{E}\left[u\left(t_{k+1}, x+\sigma \sqrt{h} \xi\right)\left(1-\frac{1}{\sigma} u^{\top}\left(t_{k+1}, x\right) \sqrt{h} \xi\right)\right]-\nabla p\left(t_{k}, x\right) h \\
& +f\left(t_{k}, x\right) h+O\left(h^{2}\right) \\
= & \frac{1}{8} \sum_{q=1}^{8} u\left(t_{k+1}, x+\sigma \sqrt{h} \xi_{q}\right)-\frac{\sqrt{h}}{\sigma} v\left(t_{k}, x\right)-\nabla p\left(t_{k}, x\right) h+f\left(t_{k}, x\right) h+O\left(h^{2}\right),
\end{aligned}
$$

where

$$
v\left(t_{k}, x\right)=\mathbb{E}\left[u\left(t_{k+1}, x+\sigma \sqrt{h} \xi\right) \xi^{\top}\right] u\left(t_{k+1}, x\right)=\frac{1}{8} \sum_{q=1}^{8} u\left(t_{k+1}, x+\sigma \sqrt{h} \xi_{q}\right) \xi_{q}^{\top} u\left(t_{k+1}, x\right) .
$$

It is useful to bear in mind that $v\left(t_{k}, x\right)=\sigma \sqrt{h}\left(u\left(t_{k+1}, x\right), \nabla\right) u\left(t_{k+1}, x\right)+O\left(h^{3 / 2}\right)$.

Using the Helmholtz-Hodge decomposition and taking into account the fact that $\operatorname{div} u\left(t_{k+1}\right.$, $\left.x+\sigma \sqrt{h} \xi_{q}\right)=0$, we obtain, from (3.33)-(3.34),

$$
\begin{aligned}
u\left(t_{k}, x\right)= & \frac{1}{8} \sum_{q=1}^{8} u\left(t_{k+1}, x+\sigma \sqrt{h} \xi_{q}\right)-\frac{\sqrt{h}}{\sigma} P v\left(t_{k}, x\right)+P f\left(t_{k}, x\right) h \\
& -\frac{\sqrt{h}}{\sigma} P^{\perp} v\left(t_{k}, x\right)+P^{\perp} f\left(t_{k}, x\right) h-\nabla p\left(t_{k}, x\right) h+O\left(h^{2}\right),
\end{aligned}
$$

whence

$$
\begin{gathered}
u\left(t_{k}, x\right)=\frac{1}{8} \sum_{q=1}^{8} u\left(t_{k+1}, x+\sigma \sqrt{h} \xi_{q}\right)-\frac{\sqrt{h}}{\sigma} P v\left(t_{k}, x\right)+P f\left(t_{k}, x\right) h+O\left(h^{2}\right), \\
\nabla p\left(t_{k}, x\right) h=-\frac{\sqrt{h}}{\sigma} P^{\perp} v\left(t_{k}, x\right)+P^{\perp} f\left(t_{k}, x\right) h+O\left(h^{2}\right) .
\end{gathered}
$$


Based on (3.35)-(3.36), we propose the following method:

$$
\begin{gathered}
\bar{u}\left(t_{N}, x\right)=\varphi(x), \\
\bar{u}\left(t_{k}, x\right)=\frac{1}{8} \sum_{q=1}^{8} \bar{u}\left(t_{k+1}, x+\sigma \sqrt{h} \xi_{q}\right)-\frac{\sqrt{h}}{\sigma} P \bar{v}\left(t_{k}, x\right)+P f\left(t_{k}, x\right) h, \\
\nabla \bar{p}\left(t_{k}, x\right) h=-\frac{\sqrt{h}}{\sigma} P^{\perp} \bar{v}\left(t_{k}, x\right)+P^{\perp} f\left(t_{k}, x\right) h, \quad k=N-1, \ldots, 0 .
\end{gathered}
$$

Here

$$
\bar{v}\left(t_{k}, x\right)=\frac{1}{8} \sum_{q=1}^{8} \bar{u}\left(t_{k+1}, x+\sigma \sqrt{h} \xi_{q}\right) \xi_{q}^{\top} \bar{u}\left(t_{k+1}, x\right) .
$$

Due to (3.35), method (3.37)-(3.38), (3.40) has the one-step error of order 2. Analogously to Proposition 3.1, it can be proved that this method converges and is of first order. As to the pressure, it can be evaluated according to (3.39), and, under the corresponding assumptions, inequality (3.27) holds and the discussion before Proposition 3.2 is also valid here.

We note that the practical realization of method (3.37)-(3.40) is simpler than that of (3.5)(3.6). Implementation of layer methods in the considered periodic case relies on the Fourier expansions (3.8). The realization of (3.5)-(3.6) requires substituting one Fourier expansion into another to compute (3.7), while evaluating (3.40) in the layer method (3.37)-(3.40) requires only multiplication of two Fourier series (see the further discussion in Section 7).

Let us discuss a relationship between layer methods and finite difference methods. For simplicity in writing, we give this illustration in the two-dimensional case. It is not difficult to note that the two-dimensional analog of the layer approximation (3.38) can be rewritten as the following finite difference scheme for the NSEs:

$$
\begin{aligned}
\frac{\bar{u}\left(t_{k}, x\right)-\bar{u}\left(t_{k+1}, x\right)}{h} & \\
= & \frac{\bar{u}\left(t_{k+1}, x^{1}+\sigma \sqrt{h}, x^{2}+\sigma \sqrt{h}\right)+\bar{u}\left(t_{k+1}, x^{1}-\sigma \sqrt{h}, x^{2}+\sigma \sqrt{h}\right)-4 \bar{u}\left(t_{k+1}, x^{1}, x^{2}\right)}{4 h} \\
& \quad+\frac{\bar{u}\left(t_{k+1}, x^{1}+\sigma \sqrt{h}, x^{2}-\sigma \sqrt{h}\right)+\bar{u}\left(t_{k+1}, x^{1}-\sigma \sqrt{h}, x^{2}-\sigma \sqrt{h}\right)}{4 h} \\
& \quad-\frac{1}{\sigma \sqrt{h}} P \bar{v}\left(t_{k}, x\right)+\operatorname{Pf}\left(t_{k}, x\right)
\end{aligned}
$$

with

$$
\begin{aligned}
\frac{\bar{v}\left(t_{k}, x\right)}{\sigma \sqrt{h}}= & \bar{u}^{1}\left(t_{k+1}, x\right) \frac{\bar{u}\left(t_{k+1}, x^{1}+\sigma \sqrt{h}, x^{2}+\sigma \sqrt{h}\right)-\bar{u}\left(t_{k+1}, x^{1}-\sigma \sqrt{h}, x^{2}+\sigma \sqrt{h}\right)}{4 \sigma \sqrt{h}} \\
& +\bar{u}^{1}\left(t_{k+1}, x\right) \frac{\bar{u}\left(t_{k+1}, x^{1}+\sigma \sqrt{h}, x^{2}-\sigma \sqrt{h}\right)-\bar{u}\left(t_{k+1}, x^{1}-\sigma \sqrt{h}, x^{2}-\sigma \sqrt{h}\right)}{4 \sigma \sqrt{h}} \\
& +\bar{u}^{2}\left(t_{k+1}, x\right) \frac{\bar{u}\left(t_{k+1}, x^{1}+\sigma \sqrt{h}, x^{2}+\sigma \sqrt{h}\right)-\bar{u}\left(t_{k+1}, x^{1}+\sigma \sqrt{h}, x^{2}-\sigma \sqrt{h}\right)}{4 \sigma \sqrt{h}} \\
& +\bar{u}^{2}\left(t_{k+1}, x\right) \frac{\bar{u}\left(t_{k+1}, x^{1}-\sigma \sqrt{h}, x^{2}+\sigma \sqrt{h}\right)-\bar{u}\left(t_{k+1}, x^{1}-\sigma \sqrt{h}, x^{2}-\sigma \sqrt{h}\right)}{4 \sigma \sqrt{h}} .
\end{aligned}
$$


As one can see, $\bar{u}\left(t_{k+1}, x\right)$ on the right-hand side of (3.41) is evaluated at the nodes $\left(x^{1}, x^{2}\right)$ and $\left(x^{1} \pm \sigma \sqrt{h}, x^{2} \pm \sigma \sqrt{h}\right)$, which is typical for a standard first-order explicit finite difference scheme with the space discretization step $h_{x}$ taken equal to $\sigma \sqrt{h}$ and $h$ being the time discretization step.

If in the weak approximation (3.32) we choose a different random vector $\xi$ taking the values

$$
\xi_{1}=(\sqrt{2}, 0)^{\top}, \quad \xi_{2}=(-\sqrt{2}, 0)^{\top}, \quad \xi_{3}=(0, \sqrt{2})^{\top}, \quad \xi_{4}=(0,-\sqrt{2})^{\top},
$$

with equal probabilities, then we obtain another first-order layer method which can be rewritten as the finite difference scheme

$$
\begin{aligned}
\frac{\bar{u}\left(t_{k}, x\right)-\bar{u}\left(t_{k+1}, x\right)}{h} & \\
= & \frac{\bar{u}\left(t_{k+1}, x^{1}+\sigma \sqrt{2 h}, x^{2}\right)+\bar{u}\left(t_{k+1}, x^{1}-\sigma \sqrt{2 h}, x^{2}\right)-4 \bar{u}\left(t_{k+1}, x^{1}, x^{2}\right)}{4 h} \\
& +\frac{\bar{u}\left(t_{k+1}, x^{1}, x^{2}+\sigma \sqrt{2 h}\right)+\bar{u}\left(t_{k+1}, x^{1}, x^{2}-\sigma \sqrt{2 h}\right)}{4 h} \\
& -\frac{1}{\sigma \sqrt{h}} P \bar{v}\left(t_{k}, x\right)+P f\left(t_{k}, x\right)
\end{aligned}
$$

with

$$
\begin{aligned}
\frac{\bar{v}\left(t_{k}, x\right)}{\sigma \sqrt{h}}= & \bar{u}^{1}\left(t_{k+1}, x\right) \frac{\bar{u}\left(t_{k+1}, x^{1}+\sigma \sqrt{2 h}, x^{2}\right)-\bar{u}\left(t_{k+1}, x^{1}-\sigma \sqrt{2 h}, x^{2}\right)}{2 \sigma \sqrt{2 h}} \\
& +\bar{u}^{2}\left(t_{k+1}, x\right) \frac{\bar{u}\left(t_{k+1}, x^{1}, x^{2}+\sigma \sqrt{2 h}\right)-\bar{u}\left(t_{k+1}, x^{1}, x^{2}-\sigma \sqrt{2 h}\right)}{2 \sigma \sqrt{2 h}} .
\end{aligned}
$$

As one can see, $\bar{u}\left(t_{k+1}, x\right)$ on the right-hand side of (3.42) is evaluated at the nodes $\left(x^{1}, x^{2}\right)$, $\left(x^{1} \pm \sigma \sqrt{2 h}, x^{2}\right)$, and $\left(x^{1}, x^{2} \pm \sigma \sqrt{2 h}\right)$, i.e. the space discretization step in (3.41) is equal to $\sigma \sqrt{2 h}$.

We recall [14], [18] that convergence theorems for layer methods (in comparison with the theory of finite difference methods) do not contain any conditions on the stability of their approximations. Using the layer methods, we do not need to a priori prescribe space nodes: they are obtained automatically depending on the choice of a probabilistic representation and a numerical scheme. We note that our results on convergence of the considered layer methods with order 1 immediately imply first-order convergence of the corresponding finite difference schemes (3.41) and (3.42).

To propose the third layer method based on the probabilistic representation (2.16)-(2.18), we observe that

$$
Q(s)=\exp \left(-\frac{1}{2 \sigma^{2}} \int_{t}^{s} u^{\top} u \mathrm{~d} s^{\prime}-\frac{1}{\sigma} \int_{t}^{s} u^{\top} \mathrm{d} W\left(s^{\prime}\right)\right) .
$$

Approximating the exponent in (3.43) by an Euler-type scheme, we obtain

$$
\bar{Q}_{k+1}=\exp \left(-\frac{h}{2 \sigma^{2}} u^{\top}\left(t_{k+1}, x\right) u\left(t_{k+1}, x\right)-\frac{\sqrt{h}}{\sigma} u^{\top}\left(t_{k+1}, x\right) \xi\right),
$$


where $\xi$ is the same as in (3.1). The resulting layer method has the form

$$
\begin{gathered}
\bar{u}\left(t_{N}, x\right)=\varphi(x), \\
\bar{u}\left(t_{k}, x\right)=P \bar{v}\left(t_{k}, x\right)+P f\left(t_{k}, x\right) h, \\
\nabla \bar{p}\left(t_{k}, x\right) h=P^{\perp} \bar{v}\left(t_{k}, x\right)+P^{\perp} f\left(t_{k}, x\right) h, \quad k=N-1, \ldots, 0,
\end{gathered}
$$

where

$$
\begin{aligned}
\bar{v}\left(t_{k}, x\right)=\frac{1}{8} \sum_{q=1}^{8} & \bar{u}\left(t_{k+1}, x+\sigma \sqrt{h} \xi_{q}\right) \\
& \times \exp \left(-\frac{h}{2 \sigma^{2}} \bar{u}^{\top}\left(t_{k+1}, x\right) \bar{u}\left(t_{k+1}, x\right)-\frac{\sqrt{h}}{\sigma} \bar{u}^{\top}\left(t_{k+1}, x\right) \xi_{q}\right)
\end{aligned}
$$

and $\xi_{1}=(1,1,1)^{\top}, \ldots, \xi_{8}=(-1,-1,-1)^{\top}$. We note that the layer method (3.45)-(3.47) can have some computational advantages in comparison with method (3.37)-(3.40) since the approximation of $Q$ by (3.44) preserves positivity for all $h$. Analogously to Proposition 3.1, we can prove that the layer method (3.45)-(3.47) is of first order.

We note that the layer method (3.45)-(3.47) as well as the layer method (3.5)-(3.7) cannot be rewritten as a finite difference scheme.

\subsection{First-order methods based on the probabilistic representation (2.20)-(2.21)}

Using the probabilistic representation (2.20)-(2.21), we consider another first-order method for NSEs. We apply the explicit Euler scheme with the simplest noise simulation applied to (2.20):

$$
\bar{X}_{t_{k}, x}\left(t_{k+1}\right)=x+\sigma \sqrt{h} \xi .
$$

Here $\xi$ is the same as in (3.1). Approximating $X_{t_{k}, x}\left(t_{k+1}\right)$ in (2.21) by $\bar{X}_{t_{k}, x}\left(t_{k+1}\right)$ from (3.48), we obtain

$$
\begin{aligned}
u\left(t_{k}, x\right)= & \mathbb{E} u\left(t_{k+1}, x+\sigma \sqrt{h} \xi\right)-\left(u\left(t_{k+1}, x\right), \nabla\right) u\left(t_{k+1}, x\right) h-\nabla p\left(t_{k}, x\right) h \\
& +f\left(t_{k}, x\right) h+O\left(h^{2}\right) \\
= & \frac{1}{8} \sum_{q=1}^{8} u\left(t_{k+1}, x+\sigma \sqrt{h} \xi_{q}\right)-\left(u\left(t_{k+1}, x\right), \nabla\right) u\left(t_{k+1}, x\right) h-\nabla p\left(t_{k}, x\right) h \\
& +f\left(t_{k}, x\right) h+O\left(h^{2}\right) .
\end{aligned}
$$

Using the Helmholtz-Hodge decomposition and taking into account the fact that $\operatorname{div} u\left(t_{k+1}, x+\right.$ $\left.\sigma \sqrt{h} \xi_{q}\right)=0$, we obtain, from (3.49),

$$
\begin{aligned}
u\left(t_{k}, x\right)= & \frac{1}{8} \sum_{q=1}^{8} u\left(t_{k+1}, x+\sigma \sqrt{h} \xi_{q}\right)-P\left(u\left(t_{k+1}, x\right), \nabla\right) u\left(t_{k+1}, x\right) h+P f\left(t_{k}, x\right) h \\
& -P^{\perp}\left(u\left(t_{k+1}, x\right), \nabla\right) u\left(t_{k+1}, x\right) h+P^{\perp} f\left(t_{k}, x\right) h-\nabla p\left(t_{k}, x\right) h+O\left(h^{2}\right),
\end{aligned}
$$

whence

$$
\begin{aligned}
u\left(t_{k}, x\right)= & \frac{1}{8} \sum_{q=1}^{8} u\left(t_{k+1}, x+\sigma \sqrt{h} \xi_{q}\right)-P\left(u\left(t_{k+1}, x\right), \nabla\right) u\left(t_{k+1}, x\right) h \\
& +P f\left(t_{k}, x\right) h+O\left(h^{2}\right), \\
\nabla p\left(t_{k}, x\right) h= & -P^{\perp}\left(u\left(t_{k+1}, x\right), \nabla\right) u\left(t_{k+1}, x\right) h+P^{\perp} f\left(t_{k}, x\right) h+O\left(h^{2}\right) .
\end{aligned}
$$


Based on (3.50)-(3.51), we propose the following method:

$$
\begin{gathered}
\bar{u}\left(t_{N}, x\right)=\varphi(x), \\
\bar{u}\left(t_{k}, x\right)=\frac{1}{8} \sum_{q=1}^{8} \bar{u}\left(t_{k+1}, x+\sigma \sqrt{h} \xi_{q}\right)-P\left(\bar{u}\left(t_{k+1}, x\right), \nabla\right) \bar{u}\left(t_{k+1}, x\right) h+P f\left(t_{k}, x\right) h, \\
\nabla \bar{p}\left(t_{k}, x\right) h=-P^{\perp}\left(\bar{u}\left(t_{k+1}, x\right), \nabla\right) \bar{u}\left(t_{k+1}, x\right) h+P^{\perp} f\left(t_{k}, x\right) h, \quad k=N-1, \ldots, 0 .
\end{gathered}
$$

Due to (3.50), the one-step error of method (3.52)-(3.53) is of second order:

$$
\begin{aligned}
& \left\|\frac{1}{8} \sum_{q=1}^{8} u\left(t_{k+1}, x+\sigma \sqrt{h} \xi_{q}\right)-P\left(u\left(t_{k+1}, x\right), \nabla\right) u\left(t_{k+1}, x\right) h+P f\left(t_{k}, x\right) h-u\left(t_{k}, x\right)\right\| \\
& \quad \leq C h^{2} .
\end{aligned}
$$

Analogously to Proposition 3.1, we prove that this method is of first order.

Proposition 3.3. Let Assumption 2.1 hold. We also assume that $\bar{u}\left(t_{k}, x\right)$ has derivatives with respect to $x$ up to some order for $x \in \mathbb{R}^{n}, k=N-1, \ldots, 0$, uniformly bounded with respect to all sufficiently small time steps $h$. Then method (3.52)-(3.53) is of first order with respect to the velocity $u$, i.e.

$$
\left\|\bar{u}\left(t_{k}, \cdot\right)-u\left(t_{k}, \cdot\right)\right\| \leq C h
$$

where the constant $C$ does not depend on $h$ and $k$.

Proof. Denote the error of method (3.52)-(3.53) on the $k$ th layer as $\varepsilon\left(t_{k}, x\right):=\bar{u}\left(t_{k}, x\right)-$ $u\left(t_{k}, x\right)$. Then (3.53) can be rewritten in the form

$$
\begin{aligned}
u\left(t_{k}, x\right)+\varepsilon\left(t_{k}, x\right)= & \frac{1}{8} \sum_{q=1}^{8} u\left(t_{k+1}, x+\sigma \sqrt{h} \xi_{q}\right)-P\left(u\left(t_{k+1}, x\right), \nabla\right) u\left(t_{k+1}, x\right) h \\
& +P f\left(t_{k}, x\right) h+\frac{1}{8} \sum_{q=1}^{8} \varepsilon\left(t_{k+1}, x+\sigma \sqrt{h} \xi_{q}\right) \\
& -P\left(\varepsilon\left(t_{k+1}, x\right), \nabla\right) \bar{u}\left(t_{k+1}, x\right) h-P\left(u\left(t_{k+1}, x\right), \nabla\right) \varepsilon\left(t_{k+1}, x\right) h .
\end{aligned}
$$

We obtain, from (3.55) and (3.57),

$$
\begin{aligned}
\varepsilon\left(t_{k}, x\right)= & \frac{1}{8} \sum_{q=1}^{8} \varepsilon\left(t_{k+1}, x+\sigma \sqrt{h} \xi_{q}\right)-P\left(\varepsilon\left(t_{k+1}, x\right), \nabla\right) \bar{u}\left(t_{k+1}, x\right) h \\
& -P\left(u\left(t_{k+1}, x\right), \nabla\right) \varepsilon\left(t_{k+1}, x\right) h+O\left(h^{2}\right)
\end{aligned}
$$

where $\left\|O\left(h^{2}\right)\right\| \leq C h^{2}$.

Introduce

$$
\varepsilon_{k}:=\left\|\varepsilon\left(t_{k}, \cdot\right)\right\| .
$$

Thanks to the assumption on the spatial derivatives of $\bar{u}\left(t_{k}, x\right)$, we obtain

$$
\left\|P\left(\varepsilon\left(t_{k+1}, \cdot\right), \nabla\right) \bar{u}\left(t_{k+1}, \cdot\right)\right\| \leq K \varepsilon_{k+1} .
$$


Using Assumption 2.1 and the assumption on the spatial derivatives of $\bar{u}\left(t_{k}, x\right)$, it is not difficult to show that

$$
\begin{aligned}
& \frac{1}{8} \sum_{q=1}^{8} \varepsilon\left(t_{k+1}, x+\sigma \sqrt{h} \xi_{q}\right)-P\left(u\left(t_{k+1}, x\right), \nabla\right) \varepsilon\left(t_{k+1}, x\right) h \\
& \quad=\frac{1}{8} \sum_{q=1}^{8} P\left[\varepsilon\left(t_{k+1}, x+\sigma \sqrt{h} \xi_{q}-u\left(t_{k+1}, x\right) h\right)\right]+O\left(h^{2}\right),
\end{aligned}
$$

where $\left|O\left(h^{2}\right)\right| \leq C h^{2}$. Analogously to (3.22) from the proof of Proposition 3.1, we obtain

$$
\left\|P\left[\varepsilon\left(t_{k+1}, x+\sigma \sqrt{h} \xi_{q}-u\left(t_{k+1}, x\right) h\right)\right]\right\| \leq \varepsilon_{k+1}\left(1+C h^{2}\right) .
$$

The error estimate (3.56) follows from (3.58), (3.59), (3.60), and (3.61). This completes the proof.

As to the pressure, it can be evaluated according to (3.54), and, under the corresponding assumptions, inequality (3.27) holds and the discussion before Proposition 3.2 is also valid here.

It is interesting to note the relationship between methods (3.37)-(3.40) and (3.52)-(3.54): $\bar{v}\left(t_{k}, x\right)$ from (3.40) is a finite difference approximation of the term $\left(\bar{u}\left(t_{k+1}, x\right), \nabla\right) \bar{u}\left(t_{k+1}, x\right) h$ in (3.52)-(3.53).

\section{Liouvillian methods}

Denote by $X_{t, x}(s)$ the phase flow of the stochastic differential equations (2.13). Let $D_{t} \in \mathbb{R}^{n}$ be a domain with finite volume. The transformation $X_{t, x}(s)$ maps $D_{t}$ into the domain $D_{s}$. The volume $V_{s}$ of the domain $D_{s}$ is equal to

$$
V_{s}=\int_{D_{s}} \mathrm{~d} X^{1} \cdots \mathrm{d} X^{n}=\int_{D_{t}}\left|\frac{D\left(X^{1}, \ldots, X^{n}\right)}{D\left(x^{1}, \ldots, x^{n}\right)}\right| \mathrm{d} x^{1} \cdots \mathrm{d} x^{n} .
$$

Then, the volume-preserving condition consists of the equality

$$
J(s):=\left|\frac{D\left(X^{1}(s), \ldots, X^{n}(s)\right)}{D\left(x^{1}, \ldots, x^{n}\right)}\right|=1 .
$$

In the case of (2.13) (see [10], [18], and [23]) we have

$$
J(s)=\exp \left(-\int_{t}^{s} \operatorname{div} u\left(s^{\prime}, X\left(s^{\prime}\right)\right) \mathrm{d} s^{\prime}\right) .
$$

Then, recalling that $\operatorname{div} u=0$, we conclude that the stochastic system (2.13) is phase volume preserving (or in other words Liouvillian). Let $X_{k}, k=0, \ldots, N, t_{k+1}-t_{k}=h_{k+1}, t_{N}=t_{0}+T$ be a numerical method for (2.13) based on the one-step approximation $\bar{X}_{t, x}(t+h)$, so that

$$
X_{0}=X(t), \quad X_{k+1}=\bar{X}_{t_{k}, X_{k}}\left(t_{k+1}\right) .
$$

It is clear that a method preserves phase volume (such methods are called Liouvillian [18], [22], [28]) if its one-step approximation satisfies the equality

$$
\left|\frac{D\left(\bar{X}^{1}, \ldots, \bar{X}^{n}\right)}{D\left(x^{1}, \ldots, x^{n}\right)}\right|=1 .
$$

Taking into account the fact that there are no constructive Liouvillian methods for (2.13) of dimension 3, we limit our consideration in this section to the two-dimensional NSEs (2.4)-(2.7). 
We apply an implicit phase area-preserving method of weak order 1 from [17] and [18] to the two-dimensional system (2.13) and obtain the one-step approximation

$$
X_{t, x}(t+h) \approx \bar{X}_{t, x}(t+h)=x-h u\left(t+h, \alpha \bar{X}^{1}+(1-\alpha) x^{1},(1-\alpha) \bar{X}^{2}+\alpha x^{2}\right)+\sigma \sqrt{h} \xi,
$$

where $\bar{X}=\left(\bar{X}^{1}, \bar{X}^{2}\right)^{\top}:=\bar{X}_{t, x}(t+h)$, the parameter $\alpha \in[0,1], \xi=\left(\xi^{1}, \xi^{2}\right)^{\top}$, and $\xi^{1}, \xi^{2}$ are i.i.d. random variables with law $\mathbb{P}\left(\xi^{i}= \pm 1\right)=\frac{1}{2}$. It is known (see, e.g. [17], [18], and the references therein) that area-preserving integrators have computational advantages in comparison with usual numerical methods in long-time simulations. Then one can expect that a layer method based on an area-preserving approximation of the associated stochastic system has some benefits in comparison, e.g. with the layer method based on the Euler scheme in Section 3.1.

Following the probabilistic approach, the area-preserving one-step approximation (4.1) leads to a layer method for the NSEs (2.4)-(2.7) of the form

$$
\begin{gathered}
\bar{u}\left(t_{N}, x\right)=\varphi(x), \\
v\left(t_{k}, x\right)=\frac{1}{4} \sum_{q=1}^{4} \bar{u}\left(t_{k+1}, \bar{X}_{q}\right)
\end{gathered}
$$

with

$$
\begin{gathered}
\bar{X}_{q}=x-h \bar{u}\left(t_{k+1}, \alpha \bar{X}_{q}^{1}+(1-\alpha) x^{1},(1-\alpha) \bar{X}_{q}^{2}+\alpha x^{2}\right)+\sigma \sqrt{h} \xi_{q}, \\
\bar{u}\left(t_{k}, x\right)=P \bar{v}\left(t_{k}, x\right)+P f\left(t_{k}, x\right) h, \\
\nabla \bar{p}\left(t_{k}, x\right) h=P^{\perp} \bar{v}\left(t_{k}, x\right)+P^{\perp} f\left(t_{k}, x\right) h, \quad k=N-1, \ldots, 0,
\end{gathered}
$$

where $\xi_{1}=(1,1)^{\top}, \ldots, \xi_{4}=(-1,-1)^{\top}$. The approximation requires the solution of the nonlinear equation (4.4) to determine the nodes at which the right-hand side of (4.3) is evaluated.

Analogously to Proposition 3.1, we can prove that the layer method (4.2)-(4.6) is of first order.

\section{Second-order method}

Let us return to the spatial periodic NSEs (2.4)-(2.7) and to the local probabilistic representation (2.13)-(2.14) of its solution which we now write in the form

$$
\begin{gathered}
\mathrm{d} X=-u(s, X) \mathrm{d} s+\sigma \mathrm{d} W(s), \quad X\left(t_{k}\right)=x, \\
\mathrm{~d} Z=-\nabla p(s, X) \mathrm{d} s+f(s, X) \mathrm{d} s, \quad Z\left(t_{k}\right)=0, \\
u\left(t_{k}, x\right)=\mathbb{E}\left[u\left(t_{k+1}, X_{t_{k}, x}\left(t_{k+1}\right)\right)+Z\left(t_{k+1}\right)\right] .
\end{gathered}
$$

System (5.1) is a system of equations with additive noise of the form

$$
\mathrm{d} Y=a(s, Y) \mathrm{d} s+\sum_{r=1}^{l} \gamma_{r} \mathrm{~d} w_{r}(s),
$$

where $Y$ is a $d$-dimensional vector, the $w_{r}(s), r=1, \ldots, l$, are independent standard Wiener processes, and the $\gamma_{r}, r=1, \ldots, l$, are constant $d$-dimensional vectors. For (5.3), let us write 
the weak Runge-Kutta method of order 2 (the one-step approximation of this method is of third order):

$$
Y_{k+1}=Y_{k}+\sum_{r=1}^{l} \gamma_{r} \eta_{r k} h^{1 / 2}+\frac{h}{2}\left[a\left(t_{k}, Y_{k}\right)+a\left(t_{k+1}, Y_{k}+\sum_{r=1}^{l} \gamma_{r} \eta_{r k} h^{1 / 2}+h a\left(t_{k+1}, Y_{k}\right)\right)\right] .
$$

Here the $\eta_{r k}, r=1, \ldots, l$, are independent random variables satisfying

$$
\mathbb{E} \eta_{r k}=\mathbb{E} \eta_{r k}^{3}=\mathbb{E} \eta_{r k}^{5}=0, \quad \mathbb{E} \eta_{r k}^{2}=1, \quad \mathbb{E} \eta_{r k}^{4}=3,
$$

e.g. they can be modeled by the law

$$
\mathbb{P}(\eta=0)=\frac{2}{3}, \quad \mathbb{P}(\eta= \pm \sqrt{3})=\frac{1}{6} .
$$

Method (5.4) slightly differs from that given in [18, p. 113].

Applying (5.4) to (5.1), we obtain

$$
\begin{aligned}
X_{k+1}= & x+\sum_{r=1}^{n} \sigma_{r} \eta_{r k} h^{1 / 2}-\frac{h}{2}\left[u\left(t_{k}, x\right)+u\left(t_{k+1}, x+\sum_{r=1}^{n} \sigma_{r} \eta_{r k} h^{1 / 2}-h u\left(t_{k+1}, x\right)\right)\right] \\
:= & X_{k+1}^{*}-\frac{h}{2} u\left(t_{k}, x\right), \\
Z_{k+1}= & -\frac{h}{2}\left[\nabla p\left(t_{k}, x\right)+\nabla p\left(t_{k+1}, x+\sum_{r=1}^{n} \sigma_{r} \eta_{r k} h^{1 / 2}-h u\left(t_{k+1}, x\right)\right)\right] \\
& +\frac{h}{2}\left[f\left(t_{k}, x\right)+f\left(t_{k+1}, x+\sum_{r=1}^{n} \sigma_{r} \eta_{r k} h^{1 / 2}-h u\left(t_{k+1}, x\right)\right)\right] \\
:= & Z_{k+1}^{*}-\frac{h}{2} \nabla p\left(t_{k}, x\right) .
\end{aligned}
$$

In (5.6)-(5.7) $n=2$ in the case of two-dimensional NSEs or $n=3$ in the case of threedimensional NSEs, the $\eta_{r k}$ are distributed due to the law (5.5), and $\sigma_{1}=(\sigma, 0)^{\top}, \sigma_{2}=(0, \sigma)^{\top}$ if $n=2$ and $\sigma_{1}=(\sigma, 0,0)^{\top}, \sigma_{2}=(0, \sigma, 0)^{\top}, \sigma_{3}=(0,0, \sigma)^{\top}$ if $n=3$.

From (5.2) and due to the fact that the one-step error of method (5.6)-(5.7) is $O\left(h^{3}\right)$, we have

$$
\begin{aligned}
u\left(t_{k}, x\right) & =\mathbb{E}\left[u\left(t_{k+1}, X_{k+1}\right)+Z_{k+1}\right]+O\left(h^{3}\right) \\
& =\mathbb{E}\left[u\left(t_{k+1}, X_{k+1}^{*}-\frac{h}{2} u\left(t_{k}, x\right)\right)+Z_{k+1}^{*}-\frac{h}{2} \nabla p\left(t_{k}, x\right)\right]+O\left(h^{3}\right) .
\end{aligned}
$$

For simplicity in writing, we limit ourselves to the case $n=2$ here. Introduce the vectors $\Lambda_{1}, \ldots, \Lambda_{9}$ corresponding to the sums $\sum_{r=1}^{2} \sigma_{r} \eta_{r k}$ under the different values of the random variables $\eta_{1 k}, \eta_{2 k}$ so that

$$
\begin{array}{lll}
\Lambda_{1}=\left[\begin{array}{l}
0 \\
0
\end{array}\right], & \Lambda_{2}=\left[\begin{array}{c}
0 \\
\sqrt{3}
\end{array}\right], & \Lambda_{3}=\left[\begin{array}{c}
0 \\
-\sqrt{3}
\end{array}\right], \\
\Lambda_{4}=\left[\begin{array}{c}
\sqrt{3} \\
0
\end{array}\right], & \Lambda_{5}=\left[\begin{array}{c}
-\sqrt{3} \\
0
\end{array}\right], & \Lambda_{6}=\left[\begin{array}{l}
\sqrt{3} \\
\sqrt{3}
\end{array}\right], \\
\Lambda_{7}=\left[\begin{array}{c}
\sqrt{3} \\
-\sqrt{3}
\end{array}\right], & \Lambda_{8}=\left[\begin{array}{c}
-\sqrt{3} \\
\sqrt{3}
\end{array}\right], & \Lambda_{9}=\left[\begin{array}{l}
-\sqrt{3} \\
-\sqrt{3}
\end{array}\right] .
\end{array}
$$


Denote the corresponding $X_{k+1}^{*}$ and $Z_{k+1}^{*}$ by $X_{k+1, q}^{*}$ and $Z_{k+1, q}^{*}, q=1, \ldots, 9$ :

$$
\begin{aligned}
X_{k+1, q}^{*}= & x+\sigma \Lambda_{q} h^{1 / 2}-\frac{h}{2} u\left(t_{k+1}, x+\sigma \Lambda_{q} h^{1 / 2}-h u\left(t_{k+1}, x\right)\right), \\
Z_{k+1, q}^{*}= & -\frac{h}{2}\left[\nabla p\left(t_{k+1}, x+\sigma \Lambda_{q} h^{1 / 2}-h u\left(t_{k+1}, x\right)\right)\right] \\
& +\frac{h}{2}\left[f\left(t_{k}, x\right)+f\left(t_{k+1}, x+\sigma \Lambda_{q} h^{1 / 2}-h u\left(t_{k+1}, x\right)\right)\right] .
\end{aligned}
$$

Their probabilities are

$$
p_{1}=\frac{4}{9}, \quad p_{2}=p_{3}=p_{4}=p_{5}=\frac{1}{9}, \quad p_{6}=p_{7}=p_{8}=p_{9}=\frac{1}{36} .
$$

Hence, we obtain, from (5.8),

$$
\begin{aligned}
u\left(t_{k}, x\right)= & \frac{4}{9}\left[u\left(t_{k+1}, X_{k+1,1}^{*}-\frac{h}{2} u\left(t_{k}, x\right)\right)+Z_{k+1,1}^{*}\right] \\
& +\frac{1}{9} \sum_{q=2}^{5}\left[u\left(t_{k+1}, X_{k+1, q}^{*}-\frac{h}{2} u\left(t_{k}, x\right)\right)+Z_{k+1, q}^{*}\right] \\
& +\frac{1}{36} \sum_{q=6}^{9}\left[u\left(t_{k+1}, X_{k+1, q}^{*}-\frac{h}{2} u\left(t_{k}, x\right)\right)+Z_{k+1, q}^{*}\right] \\
& -\frac{h}{2} \nabla p\left(t_{k}, x\right)+O\left(h^{3}\right) .
\end{aligned}
$$

In (5.9), $u\left(t_{k}, x\right)$ and $\nabla p\left(t_{k}, x\right)$ are unknowns and (5.9) is implicit with respect to $u\left(t_{k}, x\right)$.

The idea of resolving the implicitness consists in using the one-step approximation $\tilde{u}\left(t_{k}, x\right)$ of the first-order method instead of the unknown $u\left(t_{k}, x\right)$ in (5.9). So, we introduce (see (3.3))

$$
v\left(t_{k}, x\right)=\frac{1}{4} \sum_{q=1}^{4} u\left(t_{k+1}, x-u\left(t_{k+1}, x\right) h+\sigma \sqrt{h} \xi_{q}\right),
$$

where $\xi_{1}=(1,1)^{\top}, \xi_{2}=(1,-1)^{\top}, \xi_{3}=(-1,1)^{\top}$, and $\xi_{4}=(-1,-1)^{\top}$, and then (see (3.4))

$$
\tilde{u}\left(t_{k}, x\right)=P v\left(t_{k}, x\right)+P f\left(t_{k}, x\right) h .
$$

The error of $\tilde{u}\left(t_{k}, x\right)$ is of $O\left(h^{2}\right)$. Therefore, substituting $\tilde{u}\left(t_{k}, x\right)$ instead of $u\left(t_{k}, x\right)$ into the right-hand side of (5.9), we obtain the one-step approximation of third order:

$$
\begin{aligned}
u\left(t_{k}, x\right)= & \frac{4}{9}\left[u\left(t_{k+1}, X_{k+1,1}^{*}-\frac{h}{2} \tilde{u}\left(t_{k}, x\right)\right)+Z_{k+1,1}^{*}\right] \\
& +\frac{1}{9} \sum_{q=2}^{5}\left[u\left(t_{k+1}, X_{k+1, q}^{*}-\frac{h}{2} \tilde{u}\left(t_{k}, x\right)\right)+Z_{k+1, q}^{*}\right] \\
& +\frac{1}{36} \sum_{q=6}^{9}\left[u\left(t_{k+1}, X_{k+1, q}^{*}-\frac{h}{2} \tilde{u}\left(t_{k}, x\right)\right)+Z_{k+1, q}^{*}\right] \\
& -\frac{h}{2} \nabla p\left(t_{k}, x\right)+O\left(h^{3}\right) .
\end{aligned}
$$


Based on the one-step approximation (5.10), we obtain the following method of second order. Let $\bar{u}\left(t_{N}, x\right)$ and $\nabla \bar{p}\left(t_{N}, x\right)$ be determined by the initial data (2.6), i.e.

$$
\bar{u}\left(t_{N}, x\right)=\varphi(x), \quad \nabla \bar{p}\left(t_{N}, x\right)=\nabla p^{\varphi}\left(t_{N}, x\right),
$$

where $p^{\varphi}\left(t_{N}, x\right)$ is found exactly using, e.g. the equation of the form (3.23) with $\varphi$ instead of $u$. Let $\bar{u}\left(t_{k+1}, x\right)$ and $\nabla \bar{p}\left(t_{k+1}, x\right)$ for some $0<k<N$ be known. We proceed to the first-order method (3.7) and then (3.5) to first obtain

$$
\tilde{v}\left(t_{k}, x\right)=\frac{1}{4} \sum_{q=1}^{4} \bar{u}\left(t_{k+1}, x-\bar{u}\left(t_{k+1}, x\right) h+\sigma \sqrt{h} \xi_{q}\right)
$$

and then obtain

$$
\breve{u}\left(t_{k}, x\right)=P \tilde{v}\left(t_{k}, x\right)+P f\left(t_{k}, x\right) h .
$$

Following (5.10), we introduce

$$
\begin{aligned}
\breve{v}\left(t_{k}, x\right)= & \frac{4}{9}\left[\bar{u}\left(t_{k+1}, \bar{X}_{k+1,1}^{*}-\frac{h}{2} \breve{u}\left(t_{k}, x\right)\right)+\bar{Z}_{k+1,1}^{*}\right] \\
& +\frac{1}{9} \sum_{q=2}^{5}\left[\bar{u}\left(t_{k+1}, \bar{X}_{k+1, q}^{*}-\frac{h}{2} \breve{u}\left(t_{k}, x\right)\right)+\bar{Z}_{k+1, q}^{*}\right] \\
& +\frac{1}{36} \sum_{q=6}^{9}\left[\bar{u}\left(t_{k+1}, \bar{X}_{k+1, q}^{*}-\frac{h}{2} \breve{u}\left(t_{k}, x\right)\right)+\bar{Z}_{k+1, q}^{*}\right],
\end{aligned}
$$

where

$$
\begin{aligned}
\bar{X}_{k+1, q}^{*}= & x+\sigma \Lambda_{q} h^{1 / 2}-\frac{h}{2} \bar{u}\left(t_{k+1}, x+\sigma \Lambda_{q} h^{1 / 2}-h \bar{u}\left(t_{k+1}, x\right)\right), \\
\bar{Z}_{k+1, q}^{*}= & -\frac{h}{2}\left[\nabla \bar{p}\left(t_{k+1}, x+\sigma \Lambda_{q} h^{1 / 2}-h \bar{u}\left(t_{k+1}, x\right)\right)\right] \\
& +\frac{h}{2}\left[f\left(t_{k}, x\right)+f\left(t_{k+1}, x+\sigma \Lambda_{q} h^{1 / 2}-h \bar{u}\left(t_{k+1}, x\right)\right)\right] .
\end{aligned}
$$

Now it is natural (see (5.9)) to put

$$
\bar{u}\left(t_{k}, x\right)=\breve{v}\left(t_{k}, x\right)-\frac{h}{2} \nabla \bar{p}\left(t_{k}, x\right),
$$

whence

$$
\bar{u}\left(t_{k}, x\right)=P \breve{v}\left(t_{k}, x\right), \quad \frac{h}{2} \nabla \bar{p}\left(t_{k}, x\right)=P^{\perp} \breve{v}\left(t_{k}, x\right), \quad k=N-1, \ldots, 0 .
$$

Thus, heuristically, we have obtained the method of second order. Let us formulate this in the following statement without providing a rigorous proof.

Proposition 5.1. Let Assumption 2.1 hold. We also assume that $\bar{u}\left(t_{k}, x\right)$ has derivatives with respect to $x$ up to some order for $x \in \mathbb{R}^{n}, k=N-1, \ldots, 0$, uniformly bounded with respect to all sufficiently small time steps $h$. Then in the two-dimensional case method (5.11)-(5.16) is of second order for the velocity $u$ and it is of first order for the pressure $p$.

It is not difficult to also derive the second-order method in the three-dimensional $(n=3)$ case. 


\section{Small viscosity case}

The main difficulty for computational practice of NSEs is that in practical problems the viscosity $\sigma^{2}$ is very small (i.e. the Reynolds number $\operatorname{Re} \sim 1 / \sigma^{2}$ is very large) with the implication that a computational grid should have a very small spatial mesh size and a very small time step. In the small viscosity case the Itô system (2.13) is with small additive noise. For stochastic systems of this type, special weak approximations were proposed in [15]. Applying these special approximations, layer methods for the Cauchy problem for semilinear PDEs were proposed in [16]. If the solution of the Cauchy problem is regular (i.e. the solution and its derivatives are bounded uniformly in $\sigma^{2}$; see, e.g. [27] and also [18, p. 443] for situations when such an assumption holds) then it turns out that errors of these proposed methods have the form of $O\left(h^{p}+\sigma^{l} h^{q}\right)$, where $p>q, l>0$, and $h$ is a step of time discretization. Owing to the fact that the accuracy order of such methods is equal to a comparatively small $q$, they are not too complicated, while due to the large $p$ and the small factor $\sigma^{l}$ at $h^{q}$, their errors are fairly low and, therefore, these methods are highly efficient. In [16] we tested these methods on the Burgers equation with small viscosity and on the generalized KPP equation with a small parameter. The tests gave quite good results not only in regular cases but also in singular cases. In this section we exploit the results of [15] and [16] in order to construct a specific layer method for NSEs with small viscosity.

Let us use the probabilistic representation (2.13)-(2.14). Applying the Runge-Kutta weak scheme with the one-step error $O\left(h^{3}+\sigma^{2} h^{2}\right)$ (see [18, p. 196]) to system (2.13), we obtain

$$
X_{t_{k}, x}\left(t_{k+1}\right) \simeq \bar{X}_{t_{k}, x}\left(t_{k+1}\right)=x-\frac{h}{2} u\left(t_{k}, x\right)-\frac{h}{2} u\left(t_{k+1}, x-h u\left(t_{k+1}, x\right)\right)+\sigma \sqrt{h} \xi,
$$

where $\xi=\left(\xi^{1}, \xi^{2}, \xi^{3}\right)^{\top}, \xi^{1}, \xi^{2}, \xi^{3}$ are i.i.d. random variables with the law $\mathbb{P}\left(\xi^{i}= \pm 1\right)=\frac{1}{2}$ $i=1,2,3$. Besides, the equalities

$$
\begin{aligned}
\mathbb{E} \int_{t_{k}}^{t_{k+1}} \nabla p\left(s, X_{t_{k}, x}(s)\right) \mathrm{d} s & \simeq \frac{h}{2}\left(\nabla p\left(t_{k}, x\right)+\nabla p\left(t_{k+1}, x-h u\left(t_{k+1}, x\right)\right)\right), \\
\mathbb{E} \int_{t_{k}}^{t_{k+1}} f\left(s, X_{t_{k}, x}(s)\right) \mathrm{d} s & \simeq \frac{h}{2}\left(f\left(t_{k}, x\right)+f\left(t_{k+1}, x-h u\left(t_{k+1}, x\right)\right)\right)
\end{aligned}
$$

hold with the same error $O\left(h^{3}+\sigma^{2} h^{2}\right)$.

Substituting $\bar{X}_{t_{k}, x}\left(t_{k+1}\right)$ from (6.1) into (2.14) instead of $X_{t_{k}, x}\left(t_{k+1}\right)$, using (6.2) and (6.3), and evaluating the expectation exactly, we obtain

$$
\begin{aligned}
u\left(t_{k}, x\right)= & \frac{1}{8} \sum_{q=1}^{8} u\left(t_{k+1}, x-\frac{h}{2} u\left(t_{k}, x\right)-\frac{h}{2} u\left(t_{k+1}, x-h u\left(t_{k+1}, x\right)\right)+\sigma \sqrt{h} \xi_{q}\right) \\
& -\frac{h}{2}\left(\nabla p\left(t_{k}, x\right)+\nabla p\left(t_{k+1}, x-h u\left(t_{k+1}, x\right)\right)\right) \\
& +\frac{h}{2}\left(f\left(t_{k}, x\right)+f\left(t_{k+1}, x-h u\left(t_{k+1}, x\right)\right)\right)+O\left(h^{3}+\sigma^{2} h^{2}\right) .
\end{aligned}
$$

Equality (6.4) is implicit with respect to $u=u\left(t_{k}, x\right)$. Let us assume (for a while) that $u\left(t_{k+1}, x\right), \nabla p\left(t_{k}, x\right)$, and $\nabla p\left(t_{k+1}, x\right)$ are known and rewrite (6.4) in the form

$$
u=\Phi\left(u ; x, h, t_{k}\right)+O\left(h^{3}+\sigma^{2} h^{2}\right),
$$

where $\Phi$ is a known function determined by (6.4). 
Consider the equality

$$
v=\Phi\left(v ; x, h, t_{k}\right) .
$$

One can use the method of simple iteration for solving (6.5) and show that

$$
v-u=O\left(h^{3}+\sigma^{2} h^{2}\right) .
$$

If we take $u\left(t_{k+1}, x\right)$ as a null iteration $v^{(0)}$, the first iteration $v^{(1)}=\Phi\left(v^{(0)} ; x, h, t_{k}\right)$ provides the one-step error $O\left(h^{2}\right)$ only. One can show that by applying the second iteration $v^{(2)}=$ $\Phi\left(v^{(1)} ; x, h, t_{k}\right)$ we obtain

$$
v-v^{(2)}=O\left(h^{3}\right), \quad u-v^{(2)}=O\left(h^{3}+\sigma^{2} h^{2}\right) .
$$

The first iteration has a comparatively complicated form. It is not difficult to prove that if one takes the simpler expression

$$
\tilde{v}^{(1)}\left(t_{k}, x\right)=u\left(t_{k+1}, x-h u\left(t_{k+1}, x\right)\right)-h \nabla p\left(t_{k+1}, x\right)+h f\left(t_{k+1}, x\right)
$$

instead of the first iteration $v^{(1)}$ (note that to achieve the overall one-step accuracy $O\left(h^{3}+\sigma^{2} h^{2}\right)$, it is sufficient to have $\tilde{v}^{(1)}$ with accuracy $O\left(h^{2}+\sigma^{2} h\right)$ ), then the iteration

$$
\begin{aligned}
\tilde{v}^{(2)}\left(t_{k}, x\right)= & \frac{1}{8} \sum_{q=1}^{8} u\left(t_{k+1}, x-\frac{h}{2} \tilde{v}^{(1)}\left(t_{k}, x\right)-\frac{h}{2} u\left(t_{k+1}, x-h u\left(t_{k+1}, x\right)\right)+\sigma \sqrt{h} \xi_{q}\right) \\
& -\frac{h}{2}\left(\nabla p\left(t_{k}, x\right)+\nabla p\left(t_{k+1}, x-h u\left(t_{k+1}, x\right)\right)\right) \\
& +\frac{h}{2}\left(f\left(t_{k}, x\right)+f\left(t_{k+1}, x-h u\left(t_{k+1}, x\right)\right)\right)
\end{aligned}
$$

satisfies

$$
\tilde{v}\left(t_{k}, x\right)-u\left(t_{k}, x\right)=O\left(h^{3}+\sigma^{2} h^{2}\right)
$$

i.e.

$$
\begin{aligned}
u\left(t_{k}, x\right)= & \frac{1}{8} \sum_{q=1}^{8} u\left(t_{k+1}, x-\frac{h}{2} \tilde{v}^{(1)}\left(t_{k}, x\right)-\frac{h}{2} u\left(t_{k+1}, x-h u\left(t_{k+1}, x\right)\right)+\sigma \sqrt{h} \xi_{q}\right) \\
& -\frac{h}{2}\left(\nabla p\left(t_{k}, x\right)+\nabla p\left(t_{k+1}, x-h u\left(t_{k+1}, x\right)\right)\right) \\
& +\frac{h}{2}\left(f\left(t_{k}, x\right)+f\left(t_{k+1}, x-h u\left(t_{k+1}, x\right)\right)\right)+O\left(h^{3}+\sigma^{2} h^{2}\right) .
\end{aligned}
$$

It is natural that a method based on this one-step approximation is of order $O\left(h^{2}+\sigma^{2} h\right)$. Also, note that

$$
\begin{aligned}
\tilde{v}^{(1)}\left(t_{k}, x\right) & =u\left(t_{k+1}, x-h u\left(t_{k+1}, x\right)\right)-h \nabla p\left(t_{k+1}, x\right)+h f\left(t_{k+1}, x\right) \\
& =P u\left(t_{k+1}, x-h u\left(t_{k+1}, x\right)\right)+h P f\left(t_{k+1}, x\right) .
\end{aligned}
$$

Let $\bar{u}\left(t_{N}, x\right)$ and $\nabla \bar{p}\left(t_{N}, x\right)$ be determined by the initial data (2.6), i.e.

$$
\bar{u}\left(t_{N}, x\right)=\varphi(x), \quad \nabla \bar{p}\left(t_{N}, x\right)=\nabla p^{\varphi}\left(t_{N}, x\right),
$$

where $p^{\varphi}\left(t_{N}, x\right)$ is found exactly using, e.g. the equation of the form (3.23) with $\varphi$ instead of $u$. 
At the next time layers we have

$$
\bar{u}\left(t_{k}, x\right)=P \breve{v}\left(t_{k}, x\right), \quad \frac{h}{2} \nabla \bar{p}\left(t_{k}, x\right)=P^{\perp} \bar{v}\left(t_{k}, x\right), \quad k=N-1, \ldots, 0,
$$

where

$$
\begin{aligned}
\breve{v}\left(t_{k}, x\right)= & \frac{1}{8} \sum_{q=1}^{8} \bar{u}\left(t_{k+1}, x-\frac{h}{2} \breve{u}\left(t_{k}, x\right)-\frac{h}{2} \bar{u}\left(t_{k+1}, x-h \bar{u}\left(t_{k+1}, x\right)\right)+\sigma \sqrt{h} \xi_{q}\right) \\
& -\frac{h}{2} \nabla \bar{p}\left(t_{k+1}, x-h \bar{u}\left(t_{k+1}, x\right)\right)+\frac{h}{2}\left(f\left(t_{k}, x\right)+f\left(t_{k+1}, x-h \bar{u}\left(t_{k+1}, x\right)\right)\right)
\end{aligned}
$$

and

$$
\breve{u}\left(t_{k}, x\right)=P \bar{u}\left(t_{k+1}, x-h \bar{u}\left(t_{k+1}, x\right)\right)+h P f\left(t_{k+1}, x\right) .
$$

We showed that the one-step error in approximating the velocity by method (6.6)-(6.7) is $O\left(h^{3}+\sigma^{2} h^{2}\right)$, which, analogously to convergence results in previous sections, leads to the global error of order $O\left(h^{2}+\sigma^{2} h\right)$. We formulate the corresponding statement below without providing a rigorous proof.

Proposition 6.1. Let Assumption 2.1 hold, and let $\bar{u}\left(t_{k}, x\right)$ and its spatial derivatives up to a sufficiently high order be uniformly bounded. Then method (6.6)-(6.7) has the global error for the velocity $u$ estimated as

$$
\left\|\bar{u}\left(t_{k}, \cdot\right)-u\left(t_{k}, \cdot\right)\right\| \leq C \cdot\left(h^{2}+\sigma^{2} h\right)
$$

where the constant $C$ does not depend on $h, \sigma^{2}$, and $k$. For the pressure, inequality (3.27) holds.

We note that the method (6.6)-(6.7) is of first order for the velocity $u$ and, naturally, it is simpler than the second-order method (5.11)-(5.16). At the same time, for sufficiently small viscosity $\sigma^{2}$ the accuracy of method (6.6)-(6.7) can be comparable with the accuracy of (5.11)(5.16).

\section{Numerical algorithms}

Practical implementation of the layer methods (3.37)-(3.40) and (3.52)-(3.54) is straightforward and efficient. As an example, below we give such an algorithm in the case of method (3.37)-(3.40). The other layer methods proposed in this paper require a more sophisticated implementation. For this purpose, let us discuss, for instance, the first-order method (3.5)-(3.7) proposed in [1]. One can introduce the space discretization $x_{j}^{i}=j L / K, j=0,1, \ldots, K-1$, $i=1,2,3$, and complement the layer method with an interpolation (e.g. trigonometric interpolation or linear interpolation) to get the values $\bar{v}\left(t_{k}, x_{j}\right)$ at each time layer, as has been done in the implementation of layer methods for semilinear PDEs [14], [18]. Then one can accurately compute Fourier coefficients corresponding to $\bar{v}\left(t_{k}, x_{j}\right)$ (preferably for the sake of efficiency, making use of the fast Fourier transform) and project this approximate $\bar{v}$ on the divergence free space. To maintain a sufficient accuracy, this implementation apparently has comparatively large computational costs. The other possibility of implementing (3.5)-(3.7) is to expand $\bar{u}\left(t_{k+1}, x-\bar{u}\left(t_{k+1}, x\right) h+\sigma \sqrt{h} \xi_{q}\right)$ from (3.7) in $h$ around $x+\sigma \sqrt{h} \xi_{q}$. The resulting approximation will coincide with (3.52)-(3.54), whose practical realization is simple 
and efficient. At the same time, we note that further development of numerical algorithms for layer methods like (3.5)-(3.7) together with their numerical testing is needed.

The layer method (3.37)-(3.40) can be practically realized by the following algorithm which, for simplicity, we write here in the two-dimensional $(n=2)$ and zero force $(f=0)$ case. We choose a positive integer $M$ as a cutoff frequency and write the approximation solution at the time $t_{k}$ as the partial sum

$$
\bar{u}\left(t_{k}, x\right)=\sum_{n_{1}=-M}^{M-1} \sum_{n_{2}=-M}^{M-1} \bar{u}_{n}\left(t_{k}\right) \mathrm{e}^{\mathrm{i}(2 \pi / L)(\boldsymbol{n}, x)},
$$

where $\boldsymbol{n}=\left(n_{1}, n_{2}\right)^{\top}$. Furthermore, we have

$$
\frac{1}{4} \sum_{q=1}^{4} \bar{u}\left(t_{k+1}, x+\sigma \sqrt{h} \xi_{q}\right)=\sum_{n_{1}=-M}^{M-1} \sum_{n_{2}=-M}^{M-1} \bar{u}_{\boldsymbol{n}}\left(t_{k+1}\right) \mathrm{e}^{\mathrm{i}(2 \pi / L)(\boldsymbol{n}, x)} \frac{1}{4} \sum_{q=1}^{4} \mathrm{e}^{\mathrm{i}(2 \pi \sigma \sqrt{h} / L)\left(\boldsymbol{n}, \xi_{q}\right)} .
$$

Then

$$
\begin{aligned}
\bar{v}\left(t_{k}, x\right) & =\frac{1}{4} \sum_{q=1}^{4} \bar{u}\left(t_{k+1}, x+\sigma \sqrt{h} \xi_{q}\right) \xi_{q}^{\top} \bar{u}\left(t_{k+1}, x\right) \\
& =\sum_{n_{1}=-N}^{M-1} \sum_{n_{2}=-N}^{M-1} \bar{u}_{\boldsymbol{n}}\left(t_{k+1}\right) \mathrm{e}^{\mathrm{i}(2 \pi / L)(\boldsymbol{n}, x)} \frac{1}{4} \sum_{q=1}^{4} \mathrm{e}^{\mathrm{i}(2 \pi \sigma \sqrt{h} / L)\left(\boldsymbol{n}, \xi_{q}\right)} \xi_{q}^{\top} \bar{u}\left(t_{k+1}, x\right) \\
& =\sum_{n_{1}=-M}^{M-1} \sum_{n_{2}=-M}^{M-1} \check{u}_{\boldsymbol{n}}\left(t_{k}\right) \mathrm{e}^{\mathrm{i}(2 \pi / L)(\boldsymbol{n}, x)} \bar{u}\left(t_{k+1}, x\right),
\end{aligned}
$$

where

$$
\check{u}_{\boldsymbol{n}}\left(t_{k}\right):=\bar{u}_{\boldsymbol{n}}\left(t_{k+1}\right) \frac{1}{4} \sum_{q=1}^{4} \mathrm{e}^{\mathrm{i}(2 \pi \sigma \sqrt{h} / L)\left(\boldsymbol{n}, \xi_{q}\right)} \xi_{q}^{\top} .
$$

Note that $\breve{u}_{n}$ is a $2 \times 2$ matrix. Let

$$
\check{u}\left(t_{k}, x\right):=\sum_{n_{1}=-M}^{M-1} \sum_{n_{2}=-M}^{M-1} \check{u}_{\boldsymbol{n}}\left(t_{k}\right) \mathrm{e}^{\mathrm{i}(2 \pi / L)(\boldsymbol{n}, x)} .
$$

Then

$$
\bar{v}\left(t_{k}, x\right)=\breve{u}\left(t_{k}, x\right) \bar{u}\left(t_{k+1}, x\right) .
$$

We obtain the algorithm

$$
\bar{u}_{\boldsymbol{n}}\left(t_{M}\right)=\varphi_{\boldsymbol{n}}, \quad \bar{u}_{\boldsymbol{n}}\left(t_{k}\right)=\check{u}_{\boldsymbol{n}}\left(t_{k}\right)-\frac{\sqrt{h}}{\sigma}\left(P \bar{v}\left(t_{k}, x\right)\right)_{\boldsymbol{n}},
$$

where

$$
\left(P \bar{v}\left(t_{k}, x\right)\right)_{n}=\bar{v}_{\boldsymbol{n}}-\frac{\bar{v}_{\boldsymbol{n}}^{\top}\left(t_{k}\right) \boldsymbol{n}}{|\boldsymbol{n}|^{2}} \boldsymbol{n}
$$

and

$$
\bar{v}_{\boldsymbol{n}}\left(t_{k}\right)=\left(\check{u}\left(t_{k}, x\right) \bar{u}\left(t_{k+1}, x\right)\right)_{\boldsymbol{n}} ; \quad \bar{p}_{\boldsymbol{n}}\left(t_{k}\right)=\frac{\bar{v}_{\boldsymbol{n}}^{\top}\left(t_{k}\right) \boldsymbol{n}}{|\boldsymbol{n}|^{2}} \boldsymbol{n}, \quad \boldsymbol{n} \neq 0 .
$$


To find $\bar{v}_{\boldsymbol{n}}\left(t_{k}\right)$, one can either multiply two partial sums of the form (7.1) and (7.2) or exploit the fast Fourier transform in the usual fashion (see, e.g. [2]) to speed up the algorithm. Algorithm (7.3) can be viewed as analogous to spectral methods [2], [26]. It is interesting that the layer method (3.37)-(3.38), (3.40) is related, on the one hand, to a finite difference scheme (see the discussion in Section 3.3) and, on the other hand, to spectral methods.

We note that it is straightforward to practically realize the layer method (3.52)-(3.54) by an algorithm analogous to algorithm (7.3). The realization of (3.52)-(3.54) requires evaluating derivatives of the approximate solution $\bar{u}\left(t_{k+1}, x\right)$, which is not difficult to do in the considered periodic case.

We remark that the error introduced by the cutoff of frequencies in (7.1) is, in general, a major problem for any direct simulation method of NSEs (see, e.g. [2], [4], and [32]). We do not consider this problem here.

\section{Numerical experiments}

Consider the two-dimensional system of NSEs (2.4)-(2.7) with (see, e.g. [29])

$$
f(t, x)=0, \varphi(x)=\left(A \sin \frac{2 \pi \kappa x^{1}}{L} \cos \frac{2 \pi \kappa x^{2}}{L},-A \cos \frac{2 \pi \kappa x^{1}}{L} \sin \frac{2 \pi \kappa x^{2}}{L}\right)^{\top}
$$

for $\kappa \in \mathbb{Z}$ and $A \in \mathbb{R}$. It is easy to check that problem (2.4)-(2.7), (8.1) has the exact solution

$$
\begin{aligned}
& u^{1}(t, x)=A \sin \frac{2 \pi \kappa x^{1}}{L} \cos \frac{2 \pi \kappa x^{2}}{L} \exp \left(-\sigma^{2}\left(\frac{2 \pi \kappa}{L}\right)^{2}(T-t)\right), \\
& u^{2}(t, x)=-A \cos \frac{2 \pi \kappa x^{1}}{L} \sin \frac{2 \pi \kappa x^{2}}{L} \exp \left(-\sigma^{2}\left(\frac{2 \pi \kappa}{L}\right)^{2}(T-t)\right), \\
& p(t, x)=\frac{A^{2}}{4}\left(\cos \frac{4 \pi \kappa x^{1}}{L}+\cos \frac{4 \pi \kappa x^{2}}{L}\right) \exp \left(-2 \sigma^{2}\left(\frac{2 \pi \kappa}{L}\right)^{2}(T-t)\right) .
\end{aligned}
$$

As one can see, initial condition (8.1) and the zero-force result in solution (8.2) which has only those modes which are present in the initial condition. It is interesting that the new layer method (3.37)-(3.38), (3.40) also possesses this property: the approximate solution obtained by algorithm (7.3) has only those modes which are present in $\varphi(x)$.

Proposition 8.1. The approximate solution of the problem (2.4)-(2.7), (8.1) obtained by algorithm (7.3) contains only those modes which are present in the initial condition $\varphi(x)$.

Proof. For convenience, we first rewrite solution (8.2) in exponential form. To this end, let us consider the solution of (2.4)-(2.7) which can be written as the sum of four modes, i.e.

$$
\begin{aligned}
u(t, x)= & u_{\boldsymbol{n}^{1}}(t) \mathrm{e}^{\mathrm{i}(2 \pi / L)\left(\boldsymbol{n}^{1}, x\right)}+u_{\boldsymbol{n}^{1}}^{*}(t) \mathrm{e}^{-\mathrm{i}(2 \pi / L)\left(\boldsymbol{n}^{1}, x\right)}+u_{\boldsymbol{n}^{2}}(t) \mathrm{e}^{\mathrm{i}(2 \pi / L)\left(\boldsymbol{n}^{2}, x\right)} \\
& +u_{\boldsymbol{n}^{2}}^{*}(t) \mathrm{e}^{-\mathrm{i}(2 \pi / L)\left(\boldsymbol{n}^{2}, x\right)}
\end{aligned}
$$

where the asterisk denotes the complex conjugate and

$$
\begin{gathered}
\boldsymbol{n}^{1}=\kappa e_{1}, \quad \boldsymbol{n}^{2}=\kappa e_{2}, \quad u_{n^{1}}(t)=\alpha r(t) e_{2}, \quad u_{n^{2}}(t)=\beta r(t) e_{1}, \\
r(t)=A \exp \left(-\sigma^{2}\left(\frac{2 \pi \kappa}{L}\right)^{2}(T-t)\right)
\end{gathered}
$$


with

$$
e_{1}=\left[\begin{array}{l}
1 \\
1
\end{array}\right], \quad e_{2}=\left[\begin{array}{c}
1 \\
-1
\end{array}\right]
$$

We note that the orthogonality of $u_{\boldsymbol{n}^{1}}(t)$ and $\boldsymbol{n}^{1}$ and of $u_{\boldsymbol{n}^{2}}(t)$ and $\boldsymbol{n}^{2}$ implies that $u(t, x)$ from (8.3) is divergence free (see (2.3)). Furthermore, it is easy to check that $u(t, x)$ from $(8.3)$ together with the corresponding pressure $p(t, x)$ is a solution of (2.4)-(2.7) with $f=0$ and the initial condition equal to $u(T, x)$ from (8.3). One can see that $\varphi(x)$ from (8.1) coincides with $u(T, x)$ from $(8.3)$ when

$$
\alpha=\beta=\frac{1}{4 \mathrm{i}} .
$$

Suppose that the approximate velocity from algorithm (7.3) can be written in the form (8.3)(8.4), i.e.

$$
\begin{aligned}
\bar{u}\left(t_{k+1}, x\right)= & \lambda_{1} e_{2} \mathrm{e}^{\mathrm{i}(2 \pi / L)\left(n^{1}, x\right)}+\lambda_{1}^{*} e_{2} \mathrm{e}^{-\mathrm{i}(2 \pi / L)\left(\boldsymbol{n}^{1}, x\right)}+\lambda_{2} e_{1} \mathrm{e}^{\mathrm{i}(2 \pi / L)\left(\boldsymbol{n}^{2}, x\right)} \\
& +\lambda_{2}^{*} e_{1} \mathrm{e}^{-\mathrm{i}(2 \pi / L)\left(\boldsymbol{n}^{2}, x\right)}
\end{aligned}
$$

with some complex $\lambda_{1}$ and $\lambda_{2}$. Note that the initial condition in (8.1) guarantees that $\bar{u}\left(t_{N}, x\right)$ is of the form (8.5) with the appropriate $\lambda_{1}$ and $\lambda_{2}$. Recall that

$$
\bar{v}\left(t_{k}, x\right)=\frac{1}{4} \sum_{q=1}^{4} \bar{u}\left(t_{k+1}, x+\sigma \sqrt{h} \xi_{q}\right) \xi_{q}^{\top} \bar{u}\left(t_{k+1}, x\right)
$$

with $\xi_{1}=-\xi_{2}=e_{1}$ and $\xi_{3}=-\xi_{4}=e_{2}$. Introduce $a_{q}:=\xi_{q}^{\top} \bar{u}\left(t_{k+1}, x\right)$. We have

$$
\begin{aligned}
& a_{1}=-a_{2}=2 \lambda_{2} \mathrm{e}^{\mathrm{i}(2 \pi / L)\left(\boldsymbol{n}^{2}, x\right)}+2 \lambda_{2}^{*} \mathrm{e}^{-\mathrm{i}(2 \pi / L)\left(\boldsymbol{n}^{2}, x\right)}, \\
& a_{3}=-a_{4}=2 \lambda_{1} \mathrm{e}^{\mathrm{i}(2 \pi / L)\left(\boldsymbol{n}^{1}, x\right)}+2 \lambda_{1}^{*} \mathrm{e}^{-\mathrm{i}(2 \pi / L)\left(\boldsymbol{n}^{1}, x\right)} .
\end{aligned}
$$

Furthermore,

$$
\begin{aligned}
\bar{u}\left(t_{k+1}, x+\sigma \sqrt{h} \xi_{1}\right)= & \lambda_{1} e_{2} \mathrm{e}^{\mathrm{i}(2 \pi / L)\left(\boldsymbol{n}^{1}, x\right)} \mathrm{e}^{\mathrm{i} 4 \pi \sigma \sqrt{h} \kappa / L}+\lambda_{1}^{*} e_{2} \mathrm{e}^{-\mathrm{i}(2 \pi / L)\left(\boldsymbol{n}^{1}, x\right)} \mathrm{e}^{-\mathrm{i} 4 \pi \sigma \sqrt{h} \kappa / L} \\
& +\lambda_{2} e_{1} \mathrm{e}^{\mathrm{i}(2 \pi / L)\left(\boldsymbol{n}^{2}, x\right)}+\lambda_{2}^{*} e_{1} \mathrm{e}^{-\mathrm{i}(2 \pi / L)\left(\boldsymbol{n}^{2}, x\right)}, \\
\bar{u}\left(t_{k+1}, x+\sigma \sqrt{h} \xi_{2}\right)= & \lambda_{1} e_{2} \mathrm{e}^{\mathrm{i}(2 \pi / L)\left(\boldsymbol{n}^{1}, x\right)} \mathrm{e}^{-\mathrm{i} 4 \pi \sigma \sqrt{h} \kappa / L}+\lambda_{1}^{*} e_{2} \mathrm{e}^{-\mathrm{i}(2 \pi / L)\left(\boldsymbol{n}^{1}, x\right)} \mathrm{e}^{\mathrm{i} 4 \pi \sigma \sqrt{h} \kappa / L} \\
& +\lambda_{2} e_{1} \mathrm{e}^{\mathrm{i}(2 \pi / L)\left(\boldsymbol{n}^{2}, x\right)}+\lambda_{2}^{*} e_{1} \mathrm{e}^{-\mathrm{i}(2 \pi / L)\left(\boldsymbol{n}^{2}, x\right)},
\end{aligned}
$$

and we obtain

$$
\begin{aligned}
& \sum_{q=1}^{2} \bar{u}\left(t_{k+1}, x+\sigma \sqrt{h} \xi_{q}\right) \xi_{q}^{\top} \bar{u}\left(t_{k+1}, x\right) \\
& \quad=a_{1} e_{2}\left(\mathrm{e}^{\mathrm{i} 4 \pi \sigma \sqrt{h} \kappa / L}-\mathrm{e}^{-\mathrm{i} 4 \pi \sigma \sqrt{h} \kappa / L}\right)\left(\lambda_{1} \mathrm{e}^{\mathrm{i}(2 \pi / L)\left(\boldsymbol{n}^{1}, x\right)}-\lambda_{1}^{*} \mathrm{e}^{-\mathrm{i}(2 \pi / L)\left(\boldsymbol{n}^{1}, x\right)}\right) .
\end{aligned}
$$

Calculating $\sum_{q=3}^{4} \bar{u}\left(t_{k+1}, x+\sigma \sqrt{h} \xi_{q}\right) \xi_{q}^{\top} \bar{u}\left(t_{k+1}, x\right)$ analogously, we arrive at

$$
\begin{aligned}
\bar{v}\left(t_{k}, x\right)= & \left(\mathrm{e}^{\mathrm{i} 4 \pi \sigma \sqrt{h} / L}-\mathrm{e}^{-\mathrm{i} 4 \pi \sigma \sqrt{h} \kappa / L}\right) \\
& \times\left\{\left[\begin{array}{l}
1 \\
0
\end{array}\right]\left(\lambda_{1} \lambda_{2} \mathrm{e}^{\mathrm{i}(4 \pi / L) \kappa x^{1}}-\lambda_{1}^{*} \lambda_{2}^{*} \mathrm{e}^{-\mathrm{i}(4 \pi / L) \kappa x^{1}}\right)\right. \\
& \left.+\left[\begin{array}{l}
0 \\
1
\end{array}\right]\left(\lambda_{1}^{*} \lambda_{2} \mathrm{e}^{-\mathrm{i}(4 \pi / L) \kappa x^{2}}-\lambda_{1} \lambda_{2}^{*} \mathrm{e}^{\mathrm{i}(4 \pi / L) \kappa x^{2}}\right)\right\} .
\end{aligned}
$$


Clearly, $P \bar{v}\left(t_{k}, x\right)=0$ (see (2.3)), and, therefore,

$$
\begin{aligned}
\bar{u}\left(t_{k}, x\right) & =\frac{1}{4} \sum_{q=1}^{4} \bar{u}\left(t_{k+1}, x+\sigma \sqrt{h} \xi_{q}\right)-\frac{\sqrt{h}}{\sigma} P \bar{v}\left(t_{k}, x\right) \\
& =\frac{1}{4} \sum_{q=1}^{4} \bar{u}\left(t_{k+1}, x+\sigma \sqrt{h} \xi_{q}\right) .
\end{aligned}
$$

Then

$$
\begin{aligned}
\bar{u}\left(t_{k}, x\right)= & \frac{1}{4} \sum_{q=1}^{4} \bar{u}\left(t_{k+1}, x+\sigma \sqrt{h} \xi_{q}\right) \\
= & \mu\left(\lambda_{1} e_{2} \mathrm{e}^{\mathrm{i}(2 \pi / L)\left(\boldsymbol{n}^{1}, x\right)}+\lambda_{1}^{*} e_{2} \mathrm{e}^{-\mathrm{i}(2 \pi / L)\left(\boldsymbol{n}^{1}, x\right)}+\lambda_{2} e_{1} \mathrm{e}^{\mathrm{i}(2 \pi / L)\left(\boldsymbol{n}^{2}, x\right)}\right. \\
& \left.\quad+\lambda_{2}^{*} e_{1} \mathrm{e}^{-\mathrm{i}(2 \pi / L)\left(\boldsymbol{n}^{2}, x\right)}\right) \\
\mu= & \frac{1}{4}\left(\mathrm{e}^{\mathrm{i} 4 \pi \sigma \sqrt{h} \kappa / L}+\mathrm{e}^{-\mathrm{i} 4 \pi \sigma \sqrt{h} \kappa / L}\right)
\end{aligned}
$$

i.e. $\bar{u}\left(t_{k}, x\right)$ is of the form (8.5). Hence, the required property is proved.

Here we test algorithm (7.3) on example (2.4)-(2.7), (8.1). We consider the following relative errors in Fourier space for the velocity and pressure, respectively:

$$
\operatorname{err}_{u}\left(t_{k}\right):=\frac{\sum_{\boldsymbol{n}}\left|\bar{u}_{\boldsymbol{n}}\left(t_{k}\right)-u_{\boldsymbol{n}}\left(t_{k}\right)\right|}{\sum_{\boldsymbol{n}}\left|u_{\boldsymbol{n}}\left(t_{k}\right)\right|}
$$

and

$$
\operatorname{err}_{p}\left(t_{k}\right):=\frac{\sum_{\boldsymbol{n}}\left|\bar{p}_{\boldsymbol{n}}\left(t_{k}\right)-p_{\boldsymbol{n}}\left(t_{k}\right)\right|}{\sum_{\boldsymbol{n}}\left|p_{\boldsymbol{n}}\left(t_{k}\right)\right|} .
$$

The results of the tests are presented in Tables 1 and 2. We observe the first order of convergence both in the velocity and pressure. The experiment confirms the theoretically proved first order of convergence for the velocity. In the case of the pressure we conjectured first-order convergence but rigorously proved the order $2 / 3$. In the experiment we observe first order of convergence for the pressure as well. We also see that the relative errors of this algorithm are very small, even for large frequencies $\kappa$.

TABLE 1: Relative errors at $t=0$ in simulation of problem (2.4)-(2.7), (8.1) with $\sigma=0.1, \kappa=1, L=1$, and $T=3$ by algorithm (7.3) with $N=4$ and various time steps $h$. The corresponding exact solution is found due to (8.2).

\begin{tabular}{cll}
\hline$h$ & Velocity & Pressure \\
\hline 0.20 & 0.016 & 0.078 \\
0.10 & 0.0078 & 0.038 \\
0.05 & 0.0039 & 0.019 \\
0.02 & 0.0016 & 0.0074 \\
0.01 & 0.0008 & 0.004 \\
\hline
\end{tabular}


TABLE 2: Relative errors at $t=0$ in simulation of problem (2.4)-(2.7), (8.1) with $\sigma=0.01, \kappa=10$, $L=1$, and $T=2$ by algorithm (7.3) with $N=20$ and various time steps $h$. The corresponding exact solution is found due to (8.2).

\begin{tabular}{ccc}
\hline$h$ & Velocity & Pressure \\
\hline 0.20 & 0.0106 & 0.089 \\
0.10 & 0.0052 & 0.043 \\
0.05 & 0.0026 & 0.021 \\
0.02 & 0.0010 & 0.008 \\
0.01 & 0.0005 & 0.004 \\
\hline
\end{tabular}

\section{Conclusions}

In this paper we have constructed a number of new layer methods for NSEs with spatial periodic boundary conditions. We obtained new first-order layer methods, among them those which are based on different probabilistic representations than those used in [1], methods preserving the phase volume, and methods specifically designed for small viscosity. A secondorder method was also proposed. Layer methods can be viewed as alternatives to the traditional finite element, spectral, and finite difference methods. We performed some numerical tests on a simple model of laminar flow and obtained promising results. At the same time, further development of numerical algorithms and their testing are required.

\section{Acknowledgements}

This work was partially supported by the Royal Society International Joint Project grant JP091142. Part of this work was done while MVT was on study leave granted by the University of Leicester.

\section{References}

[1] Belopolskaya, Y. and Milstein, G. N. (2003). An approximation method for Navier-Stokes equations based on probabilistic approach. Statist. Prob. Lett. 64, 201-211.

[2] Canuto, C., Hussaini, M. Y., Quarteroni, A. And Zang, T. A. (1988). Spectral Methods in Fluid Dynamics. Springer, New York.

[3] Chorin, A. J. And Marsden, J. E. (2000). A Mathematical Introduction to Fluid Mechanics. Springer, New York.

[4] Dubois, T., Jauberteau, F. and Temam, R. (1999). Dynamic Multilevel Methods and the Numerical Simulation of Turbulence. Cambridge University Press.

[5] Dynkin, E. B. (1965). Markov Processes, Vols I, II. Springer, Berlin.

[6] Fletcher, C. A. J. (1996). Computational Techniques for Fluid Dynamics, Vol. I. Springer, Berlin.

[7] Fletcher, C. A. J. (1996). Computational Techniques for Fluid Dynamics, Vol. II. Springer, Berlin.

[8] Girault, V. And Raviart, P.-A. (1986). Finite Element Methods for Navier-Stokes Equations. Springer, Berlin.

[9] Gobet, E., Lemor, J.-P. And Warin, X. (2005). A regression-based Monte Carlo method to solve backward stochastic differential equations. Ann. Appl. Prob. 15, 2172-2202.

[10] Kunita, H. (1990). Stochastic Flows and Stochastic Differential Equations. Cambridge University Press.

[11] Ladyzhenskaya, O. A. (1969). The Mathematical Theory of Viscous Incompressible Flow. Gordon and Breach, New York.

[12] Ma, J. And Yong, J. (1999). Forward-backward Stochastic Differential Equations and Their Applications (Lecture Notes Math. 1702). Springer, Berlin.

[13] Majda, A. J. And Bertozzi, A. L. (2003). Vorticity and Incompressible Flow. Cambridge University Press.

[14] Milstein, G. N. (2002). The probability approach to numerical solution of nonlinear parabolic equations. Numer. Methods Partial Diff. Equat. 18, 490-522.

[15] Milstein, G. N. And Tret'yakov, M. V. (1997). Numerical methods in the weak sense for stochastic differential equations with small noise. SIAM J. Numer. Anal. 34, 2142-2167. 
[16] Milstein, G. N. and Tretyakov, M. V. (2000). Numerical algorithms for semilinear parabolic equations with small parameter based on approximation of stochastic equations. Math. Comput. 69, 237-267.

[17] Milstein, G. N. and Tretyakov, M. V. (2003). Quasi-symplectic methods for Langevin-type equations. IMA J. Numer. Anal. 23, 593-626.

[18] Milstein, G. N. And Tretyakov, M. V. (2004). Stochastic Numerics for Mathematical Physics. Springer, Berlin.

[19] Milstein, G. N. and Tretyakov, M. V. (2006). Numerical algorithms for forward-backward stochastic differential equations. SIAM J. Sci. Comput. 28, 561-582.

[20] Milstein, G. N. and Tretyakov, M. V. (2007). Discretization of forward-backward stochastic differential equations and related quasi-linear parabolic equations. IMA J. Numer. Anal. 27, 24-44.

[21] Milstein, G. N. And Tretyakov, M. V. (2012). Solving the Dirichlet problem for Navier-Stokes equations by probabilistic approach. BIT Numer. Math. 52, 141-153.

[22] Milstein, G. N., Repin, Y. M. and Tretyakov, M. V. (2002). Numerical methods for stochastic systems preserving symplectic structure. SIAM J. Numer. Anal. 40, 1583-1604.

[23] Milstein, G. N., Repin, Y. M. and Tretyakov, M. V. (2002). Symplectic integration of Hamiltonian systems with additive noise. SIAM J. Numer. Anal. 39, 2066-2088.

[24] Pardoux, É. And Peng, S. (1992). Backward stochastic differential equations and quasilinear parabolic partial differential equations. In Stochastic Partial Differential Equations and Their Applications (Lecture Notes Control Inform. Sci. 176), Springer, Berlin, pp. 200-217.

[25] Peskin, C. S. (1985). A random-walk interpretation of the incompressible Navier-Stokes equations. Commun. Pure Appl. Math. 38, 845-852.

[26] Peyret, R. (2002). Spectral Methods for Incompressible Viscous Flow. Springer, New York.

[27] SMOller, J. (1983). Shock Waves and Reaction-Diffusion Equations. Springer, New York.

[28] Suris, Y. B. (1996). Partitioned Runge-Kutta methods as phase volume preserving integrators. Phys. Lett. A 220, 63-69.

[29] Taylor, G.I. (1960). The decay of eddies in a fluid. In The Scientific Papers of Sir Geoffrey Ingram Taylor, Vol. II, ed. G. K. Batchelor, Cambridge University Press, pp. 190-192.

[30] Temam, R. (1995). Navier-Stokes Equations and Nonlinear Functional Analysis. SIAM, Philadelphia, PA.

[31] Temam, R. (2001). Navier-Stokes Equations, Theory and Numerical Analysis. AMS Chelsea, Providence, RI.

[32] Wesseling, P. (2001). Principles of Computational Fluid Dynamics. Springer, Berlin. 